\title{
Mesozooplankton grazing in the Southern California Bight. I. Population abundances and gut pigment contents
}

\author{
M. R. Landry ${ }^{1}$, W. K. Peterson ${ }^{2}$, V. L. Fagerness ${ }^{2}$ \\ ${ }^{1}$ Department of Oceanography, University of Hawaii at Manoa, 1000 Pope Road, Honolulu, Hawaii 96822, USA \\ ${ }^{2}$ School of Oceanography, University of Washington, Seattle, Washington 98195 , USA
}

\begin{abstract}
Gut pigment content of the mesozooplankton community in Santa Monica Basin, California, USA was examined on 3 winter-spring and 3 autumn cruises between October 1985 and February 1988. Mean chlorophyll concentrations for the upper $70 \mathrm{~m}$ varied from 0.30 to $0.32 \mathrm{mg} \mathrm{m}^{-3}$ for autumn cruises and from 0.35 to $1.7 \mathrm{mg} \mathrm{m} \mathrm{m}^{-3}$ for winter-spring cruises. Larger crustacean zooplankton species, particularly the copepod Calanus pacificus, were more abundant in the winter-spring, and this trend was also evident in size structure of pelagic tunicates. Gut pigments of larger, migratory taxa (C. pacificus, Metridia lucens, Pleuromamma spp., large euphausiids, and ostracods) and often some of the smaller, non-migratory forms (Clausocalanus spp., Acartia spp., and appendicularians) indicated diel periodicity in feeding intensity with highest gut pigments generally at night or in the early morning. Feeding periodicity was weakest when chlorophyll concentration and mean gut pigment content (ng chl equ. ind ${ }^{-1}$ ) was highest in the spring. The night:day ratio of community gut pigment (CGP) varied from 1.02 to 1.95 for the winter-spring period and from 1.77 to 3.39 for the autumn period. Migrating taxa explained most of the day-night difference in the winter-spring but relatively little of the difference in the autumn. Small species and developmental stages ( $<1.5 \mathrm{~mm}$ body length) dominated daytime ( 95.1 to $99.8 \%$ ) and nighttime ( 63.6 to $96.2 \%$ ) CGP during the autumn and were important, though relatively less so, in the winter-spring cruises (day 58.2 to $76.9 \%$, night 37.7 to $53.7 \%$ ). Copepods accounted for the majority of CGP in May 1986 and April 1987. Thaliaceans dominated in February 1988 and were generally more important in winter-spring than autumn, as were euphausiids. Pteropods made a significant contribution to CGP in October 1985. Appendicularians (Oikopleura sp.) were major grazers during all cruises, but their contribution to CGP surpassed that of copepods on the 3 autumn cruises.
\end{abstract}

KEY WORDS: Chlorophyll - Gut pigment - Zooplankton community structure $\cdot$ Diel grazing activity

\section{INTRODUCTION}

It is a measure of the progress that has been made in understanding the tropho-dynamics of the upper oceans in recent years that we no longer regard large suspension-feeding copepods as the quintessential consumers of phytoplankton. Over the past decade, the emphasis in this regard has shifted to smaller organisms, particularly protozoans in the nano- and microplankton size range (e.g. Capriulo \& Carpenter 1980, Landry \& Hassett 1982, Azam et al. 1983, Sherr \& Sherr 1984). Even before then, however, it was clear that other groups of macroscopic animals, most notably gelatinous zooplankton such as salps and appendicularians, had been systematically overlooked and could be important grazers of primary production under some circumstances (e.g. Madin 1974, Harbison \& Gilmer 1976, Alldredge 1981).

The distribution of grazing pressure among different kinds and sizes of primary consumers has important implications for the cycling of nutrients and energy within pelagic food webs, the transfer of energy to harvestable fisheries, and the flux of particulate organic matter out of the euphotic zone (Ryther 1969, Frost 1984, Fasham 1985, Michaels \& Silver 1988, Peinert et al. 1989). Yet most studies of zooplankton feeding under field conditions continue to focus on the rates and behaviors of individual species or well-constrained groups rather than the community as a whole. The goal of the present paper is to characterize seasonal differ- 
ences in the distribution of grazing among different taxa and sizes of mesozooplankton in the Southern California Bight using gut fluorescence of chlorophyll and its byproducts as an index of grazing on phytoplankton. In a companion paper (Landry et al. 1994, this issue), we provide the experimental bases for computing grazing rates from these gut pigment standing stocks and compare the resulting estimates of mesozooplankton community grazing to measured rates of primary production and particle flux out of the euphotic zone.

\section{MATERIALS AND METHODS}

Research site. The present results are based on field studies conducted on 6 cruises in the Southern California Bight from October 1985 to February 1988. The work was done at station SCBS $305\left(33^{\circ} 45^{\prime} \mathrm{N}, 118^{\circ} 48^{\prime} \mathrm{W}\right)$, located $40 \mathrm{~km}$ off the California coast, southeast of the city of Los Angeles (Fig. 1). Prevailing currents in the area flow from southeast to northwest parallel to bathymetric contours and the coastline (Hickey 1992). This flow, the Southern California Countercurrent, is the nearshore expression of an eddy which circulates through the Bight and is bounded offshore by the southward-flowing California Current. Despite its proximity to the coastline, SCBS 305 overlies a deep coastal basin (Santa Monica Basin) with a water depth of $905 \mathrm{~m}$. The station is, therefore, semi-isolated by currents and bathymetry from near-shore, shallow water influences. Extensive studies in the Southern California Bight over the past 2 decades have documented that concentrations of dissolved nutrients, POC, ATP, phytoplankton chlorophyll a (chl a), and primary production at SCBS 305 are typical of regional averages (e.g. Mullin 1986, Small et al. 1989).

Water-column properties. Half of our cruises occurred during winter and spring months (May 1986, April 1987, and February 1988) and half in early autumn (October 1985, 1986, and 1987). We sampled the upper-

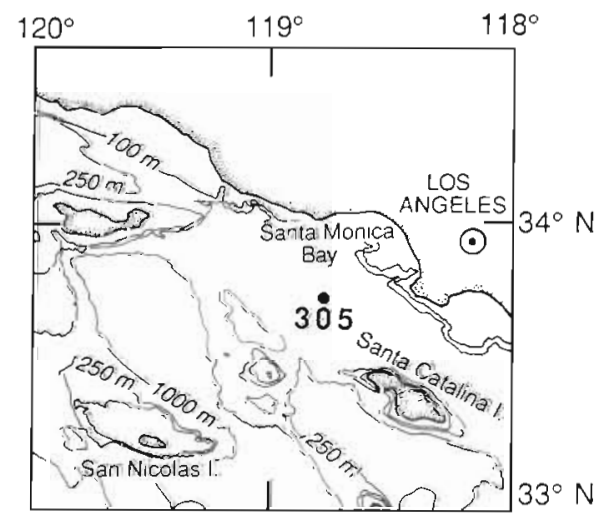

Fig. 1. Location of the sampling station SCBS 305 in the Santa Monica Basin off southern California, USA water column for temperature, salinity, chl a and phaeopigments, and zooplankton populations at $4 \mathrm{~h}$ intervals during $24 \mathrm{~h}$ deployment of a drifting sediment trap array, released at SCBS 305 before sunrise. Watercolumn sampling was conducted close to the position of the sediment traps. Depth profiles of temperature and salinity were obtained to $100 \mathrm{~m}$ with a Neil-Brown Mark III CTD. Samples for chlorophyll analysis were taken at $10 \mathrm{~m}$ depth intervals with rosette-mounted, 51 Niskin bottles with silicone rubber springs.

Pigment samples were filtered immediately on to $25 \mathrm{~mm}$ diameter Whatman GF/F glass-fiber filters. The pigments were extracted in $90 \%$ acetone using an ultrasonic cell disintegrator (Ultrasonic Megason at $300 \mathrm{~W}$ for $7 \mathrm{~min}$ ). Chl $a$ and phaeopigment concentrations were determined fluorometrically with a Turner Model 111 fluorometer (Yentsch \& Menzel 1963, HolmHansen et al. 1965).

Zooplankton abundance and gut fluorescence. Vertically integrated (70 m to surface) samples for mesozooplankton were taken with a modified bongo net frame with $215 \mu \mathrm{m}$ mesh nets. The sampler was designed such that paired $0.5 \mathrm{~m}$ net mouths faced each other in a 'closed' position during lowering to depth. When the wire angle was vertical, the net frame was open by messenger, and the nets were pulled to the sea surface at $30 \mathrm{~m} \mathrm{~min}^{-1}$. Aliquots of one of the net codends were immediately concentrated on different sizes of Nitex mesh (200 to $1000 \mu \mathrm{m})$, rinsed briefly with filtered seawater, and fast frozen on a piece of exposed metal in a $-20^{\circ} \mathrm{C}$ freezer for later determination of zooplankton gut pigment content. Occasionally, some of the larger organisms (e.g. salps, euphausiids. and copepods) were separated from the plankton sample for pigment analyses while they were still alive. Pigment contents of these organisms were found to be essentially identical to frozen specimens from the same net tow. The replicate net was thoroughly rinsed and the contents of its codend concentrated and preserved ( $4 \%$ acetate-buffered formalin) as a quantitative sample for population assessment.

Depth-integrated abundances (ind. $\mathrm{m}^{-2}$ ) of zooplankton taxa were determined from microscopical analyses of the formalin-preserved net samples. Aliquots of 2 to $4 \%$ of total sample volume were taken with a $10 \mathrm{ml}$ Stempel pipette and enumerated for small, abundant taxa. Generally these subsamples contained a hundred or more individuals each of the most dominant categories. Abundance estimates for larger, relatively rare taxa were determined by counting the entire sample.

Frozen zooplankton samples were typically analyzed for gut pigment within $24 \mathrm{~h}$ of collection. The thawed organisms were suspended in filtered seawater and sorted into taxonomic groups under a Wild dissecting 
microscope. Sorting times were variable, ranging from $5 \mathrm{~min}$ or less to 15 to $20 \mathrm{~min}$. Samples were kept in the dark except for the time when they were actually being sorted. Groups of like organisms were transferred to glass-fiber filters and ground in $90 \%$ acetone to extract pigments. The acetone extracts were analyzed for chl $a$ and phaeopigments by fluorescence as above. Larger zooplankton were removed individually from the samples using watchmaker's forceps and dipped into a dish of filtered seawater before grouping on filters. Smaller taxa were sorted and transferred to an intermediate dish of filtered water using a fine-tipped glass pipette. These organisms were then separated from residual contamination by phytoplankton and debris and placed on the glass-fiber filter by pipette with a minimal volume of water. Although large animals, e.g. salps or adult euphausiids, were sometimes analyzed individually if they were rare or variable in size, we generally measured gut pigments on groups of 10 to 20 larger specimens and 40 to 200 smaller animals. Since appendicularian 'houses' were not available from the net samples, pigment analyses for this group included only the phytoplankton material actually ingested by the organisms (e.g. Bedo et al. 1993).

Filter 'blanks' were prepared by wetting glass-fiber filters with a comparable volume of water from the rinse dish. This correction was small compared to the fluorescence measured on filters with zooplankton. 'Background' levels of individual gut fluorescence were measured as above on groups of animals starved for $24 \mathrm{~h}$ in filtered seawater. As suggested by Conover et al. (1986), we applied no correction factor to convert measured phaeopigment $(\mathrm{Ph})$ concentrations to chlorophyll equivalents (equ.). Total gut pigment content (GPC, ng pigment ind ${ }^{-1}$ ) was computed as:

$$
\mathrm{GPC}=[(\mathrm{chl} a+\mathrm{Ph}-\text { Blank }) / \mathrm{n}]-\text { Background }
$$

where $\mathrm{n}$ is the number of animals in the sample group. To normalize for temporal variability in water-column
Chlorophyll a $\left(\mathrm{mg} \mathrm{m}^{-3}\right)$
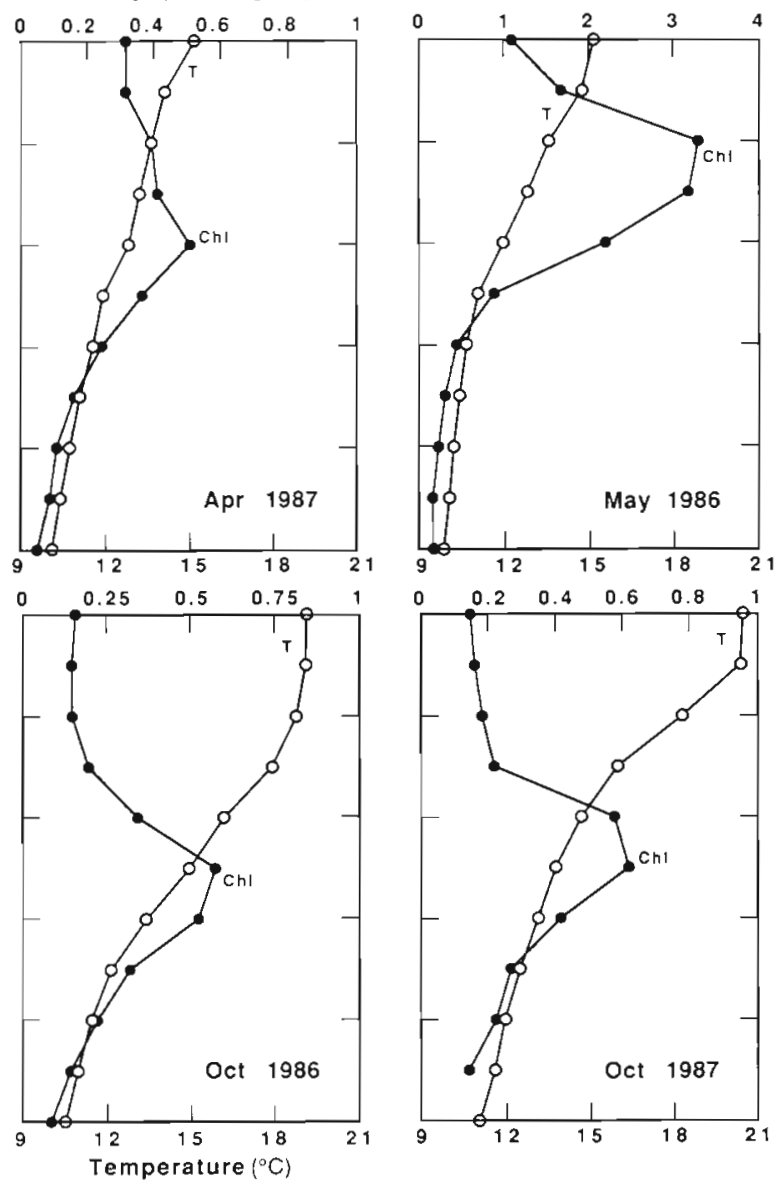

ofiles of temperature and chlorophyll a during 6
zooplankton grazing in the Santa Monica Basin

pigment, gut pigment content was converted to equivalent volume of water cleared by dividing GPC by the mean chlorophyll concentration in the top $70 \mathrm{~m}$ of the water column at the time that the zooplankton net haul was taken. Population estimates of gut pigment content (PGP, vol equ. $\mathrm{m}^{-2}$ ) were computed for cruise-averaged, daytime and nighttime periods by multiplying mean estimates of equivalent volume of water cleared per individual times population abundance (ind. $\mathrm{m}^{-2}$ ). Community gut pigment (CGP) content was determined as the sum of the equivalent volumes cleared for all component populations (i.e. individual taxa or combined groups).

\section{RESULTS}

\section{Euphotic zone}

Temperature and pigment profiles from the upper $100 \mathrm{~m}$ of the water column at SCBS 305 are compared in Fig. 2. Surface temperature ranged seasonally from 13 to $14^{\circ} \mathrm{C}$ in winter to 19 to $20^{\circ} \mathrm{C}$ in early autumn. The 
Table 1. Densities and gut pigments of mesozooplankton populations from Santa Monica Basin in October 1985. Gut pigments are expressed as equivalent (equ.) volume of mean water-column chlorophyll for the 0 to $70 \mathrm{~m}$ depth range. Population gut pigment (PGP) is the product of population density and individual pigment content. Day and night data are each the means of 3 tows. Values in parentheses indicate estimates from alternate sampling dates or times: ${ }^{\mathrm{d}}$ Oct $1986_{i}{ }^{\mathrm{b}}$ Oct $1987_{i}{ }^{\mathrm{C}} \mathrm{day}=\mathrm{night}$. Stage: $M$, male $F_{\text {, female }}$ cop., copepodids ${ }_{i} C 4(C 5)$ : copepod stage 4 (stage 5)

\begin{tabular}{|c|c|c|c|c|c|c|}
\hline \multirow[t]{2}{*}{ Taxon, stage } & \multicolumn{3}{|c|}{ Day } & \multicolumn{3}{|c|}{ Night } \\
\hline & $\begin{array}{l}\text { Density } \\
\left.\text { (ind. } \mathrm{m}^{-2}\right)\end{array}$ & $\begin{array}{l}\text { Gut pigment } \\
\left(\mathrm{ml} \text { equ. } \text { ind. }^{-1}\right)\end{array}$ & $\begin{array}{c}\text { PGP } \\
\left(1 \text { equ. } \mathrm{m}^{-2}\right)\end{array}$ & $\begin{array}{c}\text { Density } \\
\text { (ind. } \mathrm{m}^{-2} \text { ) }\end{array}$ & $\begin{array}{l}\text { Gut pigment } \\
\left(\mathrm{ml} \text { equ. ind. }{ }^{-1}\right)\end{array}$ & $\begin{array}{c}\text { PGP } \\
\text { (1 equ. } \mathrm{m}^{-2} \text { ) }\end{array}$ \\
\hline Acartia danae, $\mathrm{M} / \mathrm{F}$ & 340 & 0.49 & 0.17 & 180 & 0.80 & 0.14 \\
\hline Clausocalanus arcuicornis, $\mathrm{F}$ & 260 & 1.02 & 0.27 & 510 & 1.00 & 0.51 \\
\hline Clausocalanus pergens, $\mathrm{F}$ & 1310 & 0.34 & 0.45 & 1270 & 0.36 & 0.46 \\
\hline Small calanoids, M/F & 1430 & 0.34 & 0.49 & 2810 & 0.33 & 0.93 \\
\hline Small calanoids, cop. & 38100 & 0.16 & 6.10 & 45000 & 0.16 & 7.28 \\
\hline Oithona spp. & 36600 & $(0.10)^{\mathrm{a}}$ & 3.66 & 39800 & $(0.10)^{\mathrm{a}}$ & 3.98 \\
\hline Oncaea conifera, $\mathrm{M} / \mathrm{F}$ & 1070 & $(1.36)^{c}$ & 1.46 & 1530 & 1.36 & 2.08 \\
\hline Oncaea spp. & 15600 & $(0.46)^{3}$ & 7.18 & 22500 & $(0.78)^{a}$ & 17.55 \\
\hline Microsetella spp. & 16500 & - & - & 18100 & - & - \\
\hline Copepod nauplii & 329000 & - & - & 241000 & - & - \\
\hline Calanus pacificus, $\mathrm{F}$ & 3 & - & - & 75 & 5.22 & 0.39 \\
\hline Calanus tenuicornis, F & 0 & - & - & 32 & 6.47 & 0.21 \\
\hline Calanus spp., C4/C5 & 85 & 0.64 & 0.05 & 170 & 2.92 & 0.50 \\
\hline Eucalanus californicus, C5/F & 0 & - & - & 9 & 12.70 & 0.11 \\
\hline Metridia lucens, F & 0 & - & - & 10 & 7.30 & 0.07 \\
\hline cop. & 42 & - & - & 1020 & 3.75 & 3.82 \\
\hline Pleuromamma borealis, M/F & 0 & - & - & 30 & 2.37 & 0.07 \\
\hline Pleuromamma gracilis, M/F & 0 & - & - & 34 & 9.43 & 0.32 \\
\hline Pleuromamma spp., cop. & 0 & - & - & 345 & 2.95 & 1.02 \\
\hline Large calanoids, $>1.5 \mathrm{~mm}$ & 2 & - & - & 27 & $(6.06)^{\mathrm{b}}$ & 0.16 \\
\hline Euphausia pacifica, calyp & 760 & 0.54 & 0.41 & 850 & 0.58 & 0.49 \\
\hline $2-10 \mathrm{~mm}$ & 8 & - & - & 400 & 2.41 & 0.96 \\
\hline $10-20 \mathrm{~mm}$ & 0 & - & - & 10 & 19.30 & 0.19 \\
\hline$>20 \mathrm{~mm}$ & 0 & - & - & 5 & 39.10 & 0.20 \\
\hline Ostracods, $>1 \mathrm{~mm}$ & 0 & - & - & 130 & 33.68 & 4.38 \\
\hline Pteropods & 18800 & $(0.82)^{\mathrm{a}}$ & 15.42 & 10300 & $(0.82)^{a}$ & 8.45 \\
\hline Thaliaceans, $<1.5 \mathrm{~mm}$ & 340 & $(1.56)^{\mathrm{b}}$ & 0.53 & 170 & $(1.65)^{\mathrm{b}}$ & 0.28 \\
\hline large salps $15-40 \mathrm{~mm}$ & 2 & $(28.30)^{\mathrm{b}}$ & 0.06 & 10 & $(28.30)^{\mathrm{b}}$ & 0.28 \\
\hline Appendicularians, $<1.5 \mathrm{~mm}$ & 33400 & $(0.58)^{b}$ & 19.37 & 46100 & $(0.95)^{\mathrm{b}}$ & 43.80 \\
\hline Community gut pigment & & & 55.6 & & & 98.6 \\
\hline
\end{tabular}

winter-spring thermocline was weakly developed with a gradual, more-or-less monotonic decrease in temperature to $100 \mathrm{~m}$. By autumn, seasonal warming produced a distinct mixed-layer of 10 to $20 \mathrm{~m}$ depth overlying a sharp thermocline.

Near-surface chlorophyll concentrations varied from 0.3 to $1.1 \mathrm{mg} \mathrm{m}^{-3} \mathrm{chl}$ a for winter and spring cruises and from 0.2 to $0.3 \mathrm{mg} \mathrm{chl} \mathrm{a} \mathrm{m}^{-3}$ for summer-autumn cruises (Fig. 2). A subsurface chlorophyll maximum was always evident. Peak chlorophyll concentrations occurred at 20 to $30 \mathrm{~m}$ in February 1988 and May 1986, at $40 \mathrm{~m}$ in April 1987, and from 40 to $50 \mathrm{~m}$ in autumn. As discussed by Small et al. (1989), the maximum was generally bounded on the top by the beginning of the nitricline, the depth below which dissolved nitrate was available in measurable concentrations, and on the bottom by the depth of penetration of $1 \%$ surface irradiance. Despite obvious seasonal differences in thermal structure, zones of relatively high chlorophyll occurred between the 12 and $16^{\circ} \mathrm{C}$ isotherms on all cruises. Within this range, the chlorophyll maximum was typically present within depths bounded by the 13 and $14{ }^{\circ} \mathrm{C}$ isotherms.

Mean chlorophyll concentration in the upper $70 \mathrm{~m}$ of the water column was used for computing zooplankton gut pigments (fluorescence) in terms of equivalent volumes of water cleared of phytoplankton. This depth interval included the chlorophyll maximum on all cruises and corresponded to that for the zooplankton tows. Average concentrations of depth-integrated chlorophyll were remarkably similar for the 3 October cruises, ranging from $21.3 \mathrm{mg} \mathrm{m}^{-2}$ in 1986 to 22.7 in 1987. Mean concentrations were $0.318,0.304$, and $0.324 \mathrm{mg} \mathrm{chl} \mathrm{a} \mathrm{m} \mathrm{m}^{-3}$ for October 1985, 1986, and 1987 respectively. Within the $70 \mathrm{~m}$ depth range, pigment concentration varied within about half and double the mean values. The mean chlorophyll concentration in April 1987 (0.353 $\left.\mathrm{mg} \mathrm{m}^{-3}\right)$ was comparable to the 
Table 2. Densities and gut pigments of mesozooplankton from Santa Monica Basin in May 1986. Calculations and abbreviations as in Table 1. Values in parentheses indicate gut pigment estimates from alternate sampling dates or times of day: ${ }^{d} d a y=$ night; ${ }^{b}$ Apr 1987

\begin{tabular}{|c|c|c|c|c|c|c|}
\hline \multirow{2}{*}{ Taxon, stage } & \multicolumn{3}{|c|}{ Day } & \multicolumn{3}{|c|}{ Night } \\
\hline & $\begin{array}{l}\text { Density } \\
\text { (ind. } \mathrm{m}^{-2} \text { ) }\end{array}$ & $\begin{array}{l}\text { Gut pigment } \\
\left(\mathrm{ml} \text { equ. ind } .^{-1}\right)\end{array}$ & $\begin{array}{c}\text { PGP } \\
\left(1 \text { equ } \mathrm{m}^{-2} \text { ) }\right.\end{array}$ & $\begin{array}{l}\text { Density } \\
\text { (ind. } \mathrm{m}^{-2} \text { ) }\end{array}$ & $\begin{array}{l}\text { Gut pigment } \\
\left.\text { (ml equ ind. }{ }^{-1}\right)\end{array}$ & $\begin{array}{c}\text { PGP } \\
\left(1 \text { equ. } \mathrm{m}^{-2}\right)\end{array}$ \\
\hline Acartia hudsonsca, $\mathrm{M} / \mathrm{F}$ & 5100 & 0.41 & 2.09 & 8400 & 0.42 & 3.53 \\
\hline cop. & 14500 & 0.25 & 3.62 & 6400 & $(0.25)^{\mathrm{d}}$ & 1.60 \\
\hline Clausocalanus pergens, $\mathrm{F}$ & 1020 & 0.42 & 0.43 & 520 & 0.42 & 0.22 \\
\hline Cteno/Calo/Paracalanus, F & 4330 & 0.23 & 1.00 & 4350 & 0.48 & 2.09 \\
\hline Small calanoids, cop. & 33400 & 0.16 & 5.34 & 27900 & 0.20 & 5.58 \\
\hline Oithona similis & 6060 & $(0.25)^{\mathrm{b}}$ & 1.52 & 5600 & $(0.25)^{b}$ & 1.40 \\
\hline Oncaea spp. & 2800 & 0.63 & 1.76 & 3100 & 0.74 & 2.29 \\
\hline Copepod nauplii & 21100 & & & 44600 & & \\
\hline Calanus pacificus, $\mathrm{F}$ & 550 & 3.58 & 1.97 & 1250 & 4.28 & 5.34 \\
\hline$M$ & 250 & 0.83 & 0.20 & 270 & 1.25 & 0.34 \\
\hline $\mathrm{C} 5$ & 2770 & 2.82 & 7.81 & 3210 & 2.14 & 6.87 \\
\hline $\mathrm{C} 1-\mathrm{C} 4$ & 9450 & 0.51 & 4.82 & 12100 & 0.59 & 7.14 \\
\hline Candacia bipinnata, $\mathrm{F}$ & 60 & 3.22 & 0.19 & 50 & 1.81 & 0.09 \\
\hline Labidocera trispinosa, cop. & 0 & - & - & 250 & 0.69 & 0.17 \\
\hline Metridia lucens, $\mathrm{F}$ & 0 & - & - & 510 & 1.96 & 1.00 \\
\hline Eucalanus californicus, $\mathrm{F}$ & 760 & 7.26 & 5.52 & 150 & 7.77 & 1.17 \\
\hline Pleuromamma abdominalis, M/F & 0 & - & - & 160 & 3.35 & 0.54 \\
\hline Pleuromamma borealis, $\mathrm{M} / \mathrm{F}$ & 0 & - & - & 520 & 1.41 & 0.73 \\
\hline Pleuromamma quadrungulata & 0 & - & - & 80 & 2.33 & 0.19 \\
\hline Rhincalanus nasutus, $\mathrm{F}$ & 470 & 2.66 & 1.25 & 370 & 1.75 & 0.65 \\
\hline C5 & 100 & 1.50 & 0.15 & 140 & 1.39 & 0.19 \\
\hline Large calanoids $>1.5 \mathrm{~mm}$ & 280 & $(0.79)^{a}$ & 0.22 & 2850 & 0.79 & 2.25 \\
\hline Euphausiids, calyp & 6050 & 0.66 & 3.99 & 5510 & $(0.66)^{\mathrm{a}}$ & 3.64 \\
\hline $2-5 \mathrm{~mm}$ & 920 & 1.73 & 1.59 & 1220 & 2.67 & 3.57 \\
\hline $5-10 \mathrm{~mm}$ & 7 & - & - & 80 & 15.40 & 1.23 \\
\hline$>10 \mathrm{~mm}$ & 0 & - & - & 20 & 21.30 & 0.43 \\
\hline Ostracods, $<1.5 \mathrm{~mm}$ & 250 & 0.28 & 0.07 & 1290 & 1.15 & 1.48 \\
\hline$>1.5 \mathrm{~mm}$ & 0 & - & - & 50 & 2.85 & 0.14 \\
\hline Thaliaceans, $1-5 \mathrm{~mm}$ & 1360 & 4.62 & 6.28 & 1590 & 3.27 & 5.20 \\
\hline Appendicularians, $<1.5 \mathrm{~mm}$ & 33600 & 0.84 & 28.22 & 23500 & 0.87 & 20.44 \\
\hline Community gut pigment & & & 78.0 & & & 79.5 \\
\hline
\end{tabular}

October average, while the February 1988 mean $\left(0.726 \mathrm{mg} \mathrm{m}^{-3}\right)$ was double that for October. The highest concentration occurred in May 1986 (1.73 $\mathrm{mg} \mathrm{m}^{-3}$ ). In February and May, chlorophyll concentrations in the upper $70 \mathrm{~m}$ varied by more than an order of magnitude.

\section{Population abundances}

The abundance and composition of zooplankton populations at SCBS 305 changed seasonally. The most obvious and important change was the dramatic increase of the large copepod Calanus pacificus during winter and spring months (Tables 1 to 6). During autumn cruises, adult and late copepodids of $C$. pacificus were present at densities of 100 ind $\mathrm{m}^{-2}$ and smaller copepodid stages were rare, probably because later stages were entering diapause deeper in the water col- umn rather than reproducing. Adult densities were several thousands $\mathrm{m}^{-2}$ as early as February (Table 6), and copepodid densities remained high at least into May (Table 2). Winter and spring periods were also marked by greater abundance of other large suspension-feeding copepods, notably Eucalanus californicus, Pleuromamma spp., and Rhincalanus nasutus.

The grouping of small calanoid copepods includes adults and copepodids of Calocalanus tenuis, $C$. styliremus, Ctenocalanus vanus, Paracalanus parvus, Microcalanus, pygmaeus, Pseudocalanus sp., Acartia and Clausocalanus species, when not enumerated individually, and the small developmental stages (nominally $<1.5 \mathrm{~mm}$ ) of larger species. Clausocalanus pergens generally dominated this group and was an important component of the mesozoopankton community in all seasons. The small cyclopoids, Oithona spp. and Oncaea spp., were also abundant in all collections, but relatively more so in October. 
Table 3. Densities and gut pigment of mesozooplankton from Santa Monica Basin in October 1986. Calculations and abbreviations as in Table 1. Values in parentheses indicate estimates from alternate sampling dates or times: ${ }^{\text {act }} 1985 ;{ }^{b}$ day $=$ night

\begin{tabular}{|c|c|c|c|c|c|c|}
\hline \multirow[t]{2}{*}{ Taxon, stage } & \multicolumn{3}{|c|}{ Day } & \multicolumn{3}{|c|}{ Night } \\
\hline & $\begin{array}{l}\text { Density } \\
\text { (ind. } \mathrm{m}^{-2} \text { ) }\end{array}$ & $\begin{array}{l}\text { Gut pigment } \\
\left(\mathrm{ml} \text { equ. ind } .^{-1}\right)\end{array}$ & $\begin{array}{c}\text { PGP } \\
\left(1 \text { equ. } \mathrm{m}^{-2}\right)\end{array}$ & $\begin{array}{l}\text { Density } \\
\text { (ind. } \mathrm{m}^{-2} \text { ) }\end{array}$ & $\begin{array}{l}\text { Gut pigment } \\
\left(\mathrm{ml} \text { equ. ind }{ }^{-1}\right)\end{array}$ & $\begin{array}{c}\text { PGP } \\
\left(1 \text { equ. } \mathrm{m}^{-2}\right)\end{array}$ \\
\hline Clausocalanus arcuicornis, $\mathrm{F}$ & 790 & 0.68 & 0.54 & 1080 & 1.63 & 1.76 \\
\hline Clausocalanus pergens, $\mathrm{F}$ & 7510 & 0.19 & 1.43 & 10300 & 0.61 & 6.28 \\
\hline Clausocalanus spp., cop. & 96600 & 0.10 & 9.66 & 117800 & 0.48 & 56.54 \\
\hline Oithona similis & 43100 & 0.10 & 4.31 & 52500 & 0.10 & 5.25 \\
\hline Oithona spinirostris & 2470 & 0.48 & 1.19 & 3010 & 0.73 & 2.20 \\
\hline Oncaea spp. & 46300 & 0.46 & 21.30 & 41200 & 0.78 & 32.10 \\
\hline Microsetella spp. & 33600 & - & - & 22700 & - & - \\
\hline Copepod nauplii & 51900 & - & - & 122400 & - & - \\
\hline Calanus pacificus, $\mathrm{F}$ & 32 & 0.81 & 0.03 & 145 & 7.11 & 1.03 \\
\hline Candacia spp, , F & 2 & - & - & 14 & 10.50 & 0.15 \\
\hline Eucalanus californicus & 2 & - & - & 9 & 47.50 & 0.43 \\
\hline Metridia lucens, $\mathrm{F}$ & 7 & - & - & 202 & 7.48 & 1.51 \\
\hline cop. & 1050 & - & - & 2470 & $(3.75)^{\mathrm{a}}$ & 9.26 \\
\hline Pleuromamma borealis & 0 & - & - & 131 & 3.52 & 0.46 \\
\hline Pleuromamma gracilis & 0 & - & - & 60 & 12.60 & 0.76 \\
\hline Large calanoids, $>1.5 \mathrm{~mm}$ & 1164 & 0.41 & 0.48 & 700 & $(0.41)^{b}$ & 0.29 \\
\hline Euphausiids, calyp & 90 & $(0.54)^{\mathrm{a}}$ & 0.05 & 510 & $(0.58)^{\mathrm{a}}$ & 0.30 \\
\hline $2-5 \mathrm{~mm}$ & 51 & 0.18 & 0.01 & 64 & 4.37 & 0.28 \\
\hline $5-10 \mathrm{~mm}$ & 0 & - & - & 13 & 19.50 & 0.25 \\
\hline Ostracods $<1.5 \mathrm{~mm}$ & 85 & 0.32 & 0.03 & 260 & 13.90 & 3.61 \\
\hline$>1.5 \mathrm{~mm}$ & 0 & - & - & 23 & 91.30 & 2.10 \\
\hline Pteropods & 85 & 0.82 & 0.07 & 340 & $(0.82)^{b}$ & 0.28 \\
\hline Thaliaceans, $<2 \mathrm{~mm}$ & 430 & 0.79 & 0.34 & 420 & 8.96 & 3.76 \\
\hline Appendicularians, $<1.5 \mathrm{~mm}$ & 19900 & 1.82 & 36.22 & 23100 & 3.33 & 76.92 \\
\hline$>1.5 \mathrm{~mm}$ & 41 & $(10.30)^{b}$ & 0.42 & 32 & 10.30 & 0.33 \\
\hline Community gut pigment & & & 76.1 & & & 205.8 \\
\hline
\end{tabular}

Pteropods were not present in significant numbers in spring collections (April and May) but occurred on other cruises and were particularly abundant $\left(>10^{4}\right.$ ind. $\mathrm{m}^{-2}$ ) in October 1985 (Tables 1 to 6$)$. Ostracods, euphausiids, and thaliaceans were more prevalent in late winter and early spring. The thaliaceans category included doliolids, but it was generally dominated by small Thalia sp. Larger salp species were observed in October 1985, October 1987, and February 1988. Appendicularians (Oikopleura spp.) were numerically important in the zooplankton community during all cruises with densities characteristically in excess of $10^{4}$ ind. $\mathrm{m}^{-2}$.

The increasing importance of smaller copepod species in autumn was evident in the size structure of other zooplankton groups. The median trunk length (formalin preserved) of Oikopleura spp., for instance, ranged from 0.28 to $0.44 \mathrm{~mm}$ for October cruises and from 0.48 to 0.52 for spring and winter cruises (Fig. 3). On average, $94 \%$ of the thaliaceans were $<1.5 \mathrm{~mm}$ in October and only $0.1 \%$ were $>2.5 \mathrm{~mm}$. In contrast, $77 \%$ were $<1.5 \mathrm{~mm}$ (range 69 to $87 \%$ ) for the winterspring cruises and $9 \%$ (2 to $12 \%$ ) were $>2.5 \mathrm{~mm}$. Larger species were up to $30 \mathrm{~mm}$ in size.

\section{Gut pigments of individual taxa}

The various species, sizes, and developmental stages of zooplankton were not equally abundant on all cruises and so were grouped in different ways to achieve community estimates of gut pigment content. Nonetheless, several taxa were investigated in sufficient detail to provide diel and seasonal comparisons of gut pigment patterns. Adult females of Calanus pacificus, for instance, generally showed higher gut pigment contents at night (Fig. 4a). Nighttime gut fluorescence varied typically in the range of 5 to $10 \mathrm{ml}$ equ. water cleared ind $^{-1}$, while daytime values were usually below $5 \mathrm{ml}$ equ. ind ${ }^{-1}$ Only for May 1986, the period of highest water-column chlorophyll concentration, was this diel difference equivocal, although even then lowest gut pigment occurred in late afternoon, prior to sunset.

Developmental stages of Calanus spp. were abundant enough in April 1987 to be analyzed individually (Fig. 5). During this cruise, gut pigment contents of migratory stages ( $\mathrm{C} 4, \mathrm{C} 5$, and adult) were about a factor of 3 higher at night than in the day. Nonmigratory stages (early copepodids and nauplii) also showed a nocturnal increase in gut pigment, but only about 
Table 4. Densities and gut pigments of mesozooplankton from Santa Monica Basin in April 1987. Calculations and abbreviations

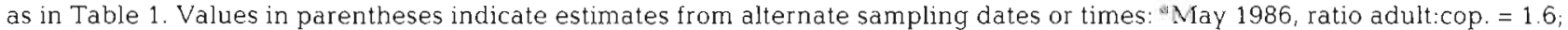
${ }^{\mathrm{b}}$ May 1986, $\mathrm{M}=0.25 \mathrm{~F} ;{ }^{\mathrm{c}} \mathrm{day}=\mathrm{night}_{;}{ }^{\mathrm{d}}$ mean of Oct 1985 and May 1986; ${ }^{\mathrm{e}}$ mean of Oct 1986 and $1987 ;{ }^{\mathrm{i}} \mathrm{Feb} 1988$

\begin{tabular}{|c|c|c|c|c|c|c|}
\hline \multirow[t]{2}{*}{ Taxon, stage } & \multicolumn{3}{|c|}{ Day } & \multicolumn{3}{|c|}{ Night } \\
\hline & $\begin{array}{l}\text { Density } \\
\text { (ind. } \mathrm{m}^{-2} \text { ) }\end{array}$ & $\begin{array}{l}\text { Gut pigment } \\
\left.\text { (m] equ. ind. }{ }^{-1}\right)\end{array}$ & $\begin{array}{c}\text { PGP } \\
\left(1 \mathrm{equ} \cdot \mathrm{m}^{-2}\right)\end{array}$ & $\begin{array}{c}\text { Density } \\
\left(\text { ind. } \mathrm{m}^{-2}\right)\end{array}$ & $\begin{array}{l}\text { Gut pigment } \\
\left(\mathrm{ml} \text { equ. ind }{ }^{-1}\right)\end{array}$ & $\begin{array}{c}\text { PGP } \\
\left(1 \text { equ. } \mathrm{m}^{-2} \text { ) }\right.\end{array}$ \\
\hline Acartia clausii, M/F & 1610 & $(1.47)^{a}$ & 2.37 & 1700 & 2.80 & 4.76 \\
\hline cop. & 5100 & 0.92 & 4.69 & 5950 & 2.71 & 16.12 \\
\hline Small calanoids, $F$ & 14300 & 1.18 & 16.87 & 12200 & 0.67 & 8.17 \\
\hline cop. & 40300 & 0.56 & 22.57 & 39000 & 0.57 & 22.23 \\
\hline Oithona spp. & 15200 & 0.25 & 3.80 & 11000 & $(0.25)^{c}$ & 2.75 \\
\hline Oncaea spp. & 7400 & 0.54 & 4.00 & 7810 & 0.58 & 4.53 \\
\hline Copepod nauplii & 11400 & - & - & 40200 & - & - \\
\hline Calanus pacificus, F & 650 & 2.78 & 1.81 & 6460 & 8.25 & 53.30 \\
\hline$M$ & 1200 & 0.70 & 0.84 & 5950 & $(2.06)^{b}$ & 12.26 \\
\hline $\mathrm{C} 4-\mathrm{C} 5$ & 6120 & 0.97 & 5.94 & 18000 & 3.89 & 70.02 \\
\hline $\mathrm{C} 1-\mathrm{C} 3$ & 32200 & 0.65 & 20.93 & 29100 & 0.85 & 24.74 \\
\hline N5-N6 & 8300 & 0.38 & 3.15 & 12400 & 0.60 & 7.44 \\
\hline Eucalanus californicus, C5/F & 16 & 25.51 & 0.41 & 0 & - & - \\
\hline Rhincalanus nasutus, C5/F & 680 & 2.12 & 1.44 & 860 & 12.03 & 10.35 \\
\hline Large calanoids, $>1.5 \mathrm{~mm}$ & 3890 & 1.24 & 4.82 & 5030 & 1.66 & 8.35 \\
\hline Euphausiids, calyp & 1910 & $(0.60)^{d}$ & 1.15 & 1530 & $(0.60)^{d}$ & 0.92 \\
\hline $2-5 \mathrm{~mm}$ & 580 & $(0.38)^{e}$ & 0.22 & 1540 & 3.48 & 5.36 \\
\hline $5-10 \mathrm{~mm}$ & 16 & $(1.40)^{6}$ & 0.02 & 140 & 9.17 & 1.28 \\
\hline$>10 \mathrm{~mm}$ & 0 & - & - & 2 & $(25.00)^{\prime}$ & 0.05 \\
\hline Ostracods,$<1.5 \mathrm{~mm}$ & 780 & 0.88 & 0.69 & 860 & 1.45 & 1.24 \\
\hline$>1.5 \mathrm{~mm}$ & 0 & - & & 8 & 5.46 & 0.04 \\
\hline Cladocera (Podon spp.) & 5860 & 0.35 & 2.05 & 6280 & 0.34 & 2.14 \\
\hline Thaliaceans, $<2 \mathrm{~mm}$ & 3590 & 2.94 & 10.56 & 2290 & $(2.94)^{\mathrm{c}}$ & 6.72 \\
\hline Appendicularians & 29000 & 1.94 & 56.26 & 24100 & 1.94 & 46.75 \\
\hline Community gut pigment & & & 164.6 & & & 320.9 \\
\hline
\end{tabular}

$50 \%$ above daytime levels. The gut pigment contents of developmental stages of $C$. pacificus relative to that for adult females were consistent with results of Hansen et al. (1990) for C. finmarchicus.
Smaller, nonmigratory zooplankton exhibited daynight differences in gut fluorescence to varying extents (Fig. 6). Nighttime values for 2 species of Clausocalanus, $C$. pergens and $C$. arcuicornis, were, on aver-
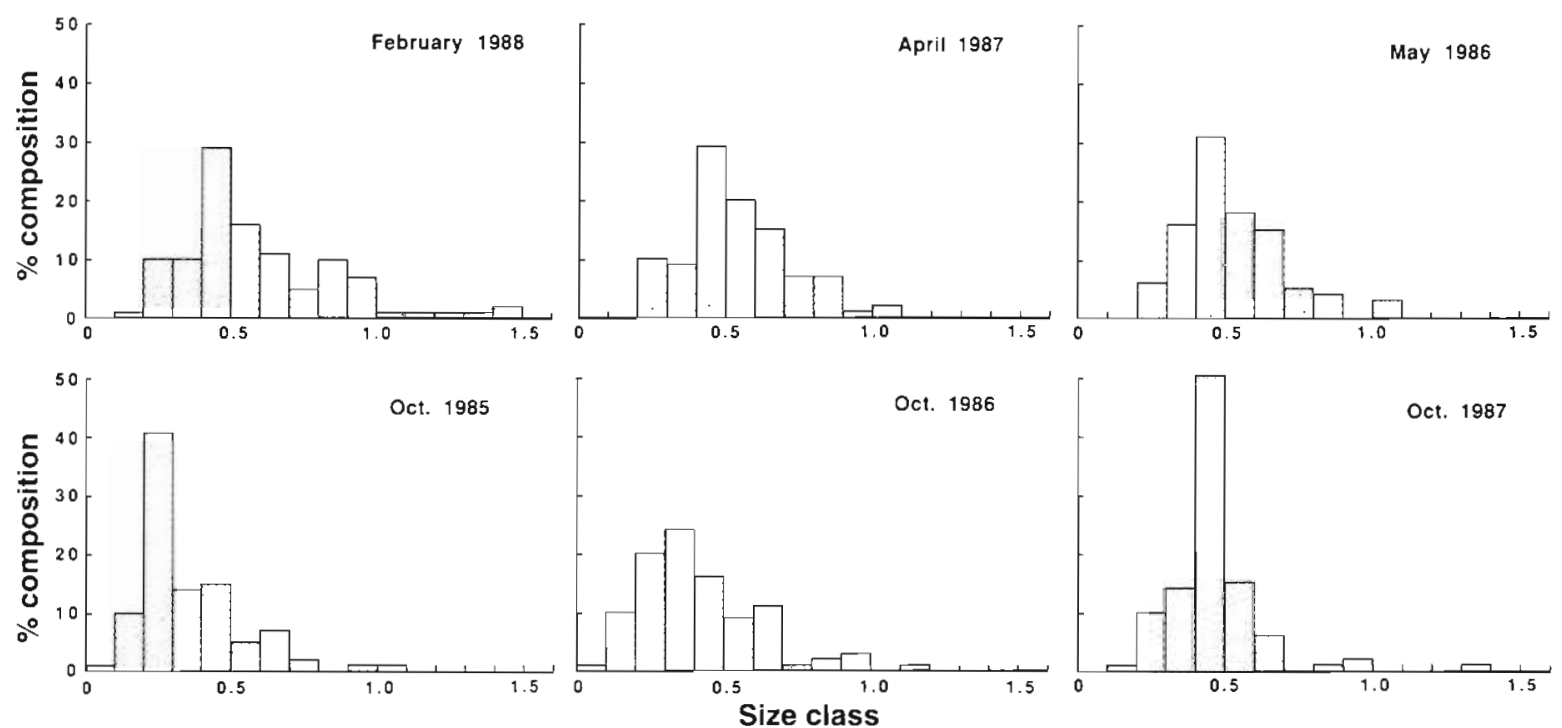

Fig. 3. Oikopleura spp. Size-frequency distribution of appendicularians sampled on 6 cruises in the Santa Monica Basin 
Table 5. Densities and gut pigments of zooplankton from Santa Monica Basin in October 1987. Calculations and abbreviations as in Table 1. Values in parentheses indicate estimates from alternate sampling dates or times: ${ }^{d}$ Oct $1986 ;{ }^{b}$ Oct $1985 ;{ }^{c}$ mean Oct 1985 and $1986 ;{ }^{d}$ Apr $1987 ;{ }^{e}$ Feb 1988

\begin{tabular}{|c|c|c|c|c|c|c|}
\hline \multirow[t]{2}{*}{ Taxon, stage } & \multicolumn{3}{|c|}{ Day } & \multicolumn{3}{|c|}{ Night } \\
\hline & $\begin{array}{l}\text { Density } \\
\text { (ind. } \mathrm{m}^{-2} \text { ) }\end{array}$ & $\begin{array}{l}\text { Gut pigment } \\
\left(\mathrm{ml} \text { equ. ind }{ }^{-1}\right)\end{array}$ & $\begin{array}{c}\text { PGP } \\
\left(1 \text { equ } m^{-2}\right)\end{array}$ & $\begin{array}{c}\text { Density } \\
\text { (ind. } \mathrm{m}^{-2} \text { ) }\end{array}$ & $\begin{array}{l}\text { Gut pigment } \\
\left.\text { (ml equ. ind } .^{-1}\right)\end{array}$ & $\begin{array}{c}\text { PGP } \\
\text { (1 equ. } \mathrm{m}^{-2} \text { ) }\end{array}$ \\
\hline Clausocalanus spp., F & 9690 & 0.26 & 2.52 & 12100 & $(0.48)^{\mathrm{a}}$ & 5.81 \\
\hline Small calanoids & 41400 & 0.12 & 4.97 & 48300 & 0.19 & 9.18 \\
\hline Oithona spp. & 13800 & $(0.10)^{\mathrm{d}}$ & 1.38 & 14700 & $(0.10)^{a}$ & 1.47 \\
\hline Oncaea spp. & 3320 & $(0.46)^{a}$ & 1.53 & 13500 & $(0.78)^{\mathrm{a}}$ & 10.53 \\
\hline Copepod nauplii & 640 & - & - & 760 & - & - \\
\hline Calanus spp., F & 360 & $(0.81)^{\mathrm{a}}$ & 0.29 & 330 & 13.26 & 4.38 \\
\hline Metridia lucens, $\mathrm{F}$ & 0 & - & - & 1070 & 8.86 & 9.48 \\
\hline Pleuromamma abdominalis, $\mathrm{F}$ & 0 & - & - & 60 & 10.76 & 0.65 \\
\hline Pleuromamma borealis, $\mathrm{F}$ & 2 & - & - & 840 & 6.45 & 5.42 \\
\hline Rhincalanus nasutus, C5/F & 10 & 1.01 & 0.01 & 15 & $(6.21)^{\mathrm{e}}$ & 0.09 \\
\hline Large calanoids, $>1.5 \mathrm{~mm}$ & 1190 & $(1.24)^{\mathrm{d}}$ & 1.48 & 3100 & 6.06 & 18.79 \\
\hline Euphausiids, calyp & 1150 & $(0.54)^{\mathrm{b}}$ & 0.62 & 890 & $(0.58)^{b}$ & 0.52 \\
\hline $2-5 \mathrm{~mm}$ & 260 & 0.57 & 0.15 & 490 & 10.56 & 5.17 \\
\hline $5-10 \mathrm{~mm}$ & 0 & - & - & 26 & $(10.96)^{c}$ & 0.28 \\
\hline$>10 \mathrm{~mm}$ & 0 & - & - & 12 & $(19.30)^{b}$ & 0.23 \\
\hline Ostracods, $<1.5 \mathrm{~mm}$ & 200 & $(0.32)^{\mathrm{a}}$ & 0.08 & 640 & $(14.08)^{\mathrm{C}}$ & 9.01 \\
\hline$>1.5 \mathrm{~mm}$ & 0 & - & - & 23 & $(62.49)^{\mathrm{c}}$ & 1.44 \\
\hline Pteropods & 640 & $(0.82)^{\mathrm{d}}$ & 0.52 & 1910 & $(0.82)^{a}$ & 1.57 \\
\hline Thaliaceans, $2-5 \mathrm{~mm}$ & 765 & 1.56 & 1.19 & 895 & 1.65 & 1.48 \\
\hline $5-10 \mathrm{~mm}$ & 0 & - & - & 5 & 7.47 & 0.04 \\
\hline$-20 \mathrm{~mm}$ & 0 & - & - & 5 & 28.30 & 0.14 \\
\hline Appendicularians, $<1.5 \mathrm{~mm}$ & 52800 & 0.58 & 30.62 & 53200 & 0.95 & 50.54 \\
\hline$>1.5 \mathrm{~mm}$ & 100 & 3.07 & 0.31 & 640 & 28.90 & 18.50 \\
\hline Community gut pigment & & & 45.7 & & & 154.7 \\
\hline
\end{tabular}

age, about double the values observed during daytime collections. In both cases, however, high values were noted during mid-afternoon on the October 1985 cruise. No diel difference in pigment content was observed for the May 1986 cruise, during which the Clausocalanus spp. were analyzed jointly. Oncaea spp. appeared to follow a similar pattern showing a daynight difference in autumn (October 1986) but none in spring (May 1986, April 1987). Appendicularians did not show evidence of a nocturnal increase in gut pigments; if anything, gut fluorescence during the autumn cruises appeared to be higher in the morning than in the evening to midnight period when copepods were most active.

Gut fluorescence of Calanus pacificus females from combined nighttime samples varied strongly with water-column chlorophyll (Fig. 4b). Gut pigment increased more or less linearly with mean chlorophyll
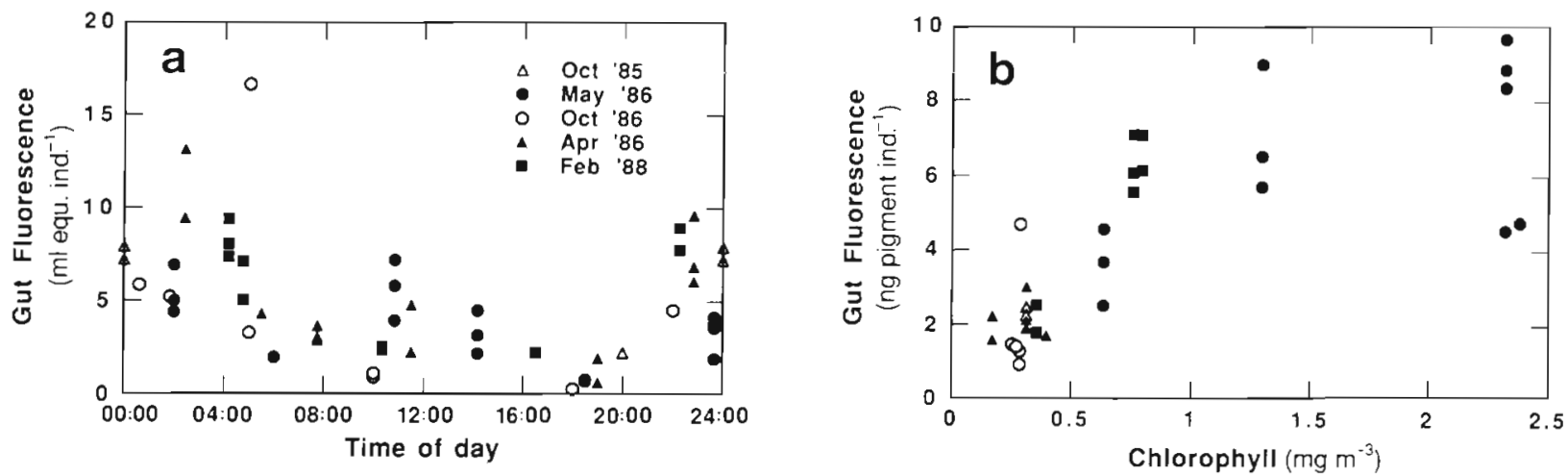

Fig. 4. Calanus pacificus. Gut pigment contents of adult females as functions of (a) time of day and (b) mean euphotic zone chlorophyll concentration 
Table 6. Densities and gut pigments of mesozooplankton from Santa Monica Basin in February 1988. Calculations and abbreviations as in Table $1 .{ }^{a}$ Assumes day and night gut pigments are equal

\begin{tabular}{|c|c|c|c|c|c|c|}
\hline \multirow[t]{2}{*}{ Taxon, stage } & \multicolumn{3}{|c|}{ Day } & \multicolumn{3}{|c|}{ Night } \\
\hline & $\begin{array}{l}\text { Density } \\
\text { (ind. } \mathrm{m}^{-2} \text { ) }\end{array}$ & $\begin{array}{l}\text { Gut pigment } \\
\left.\text { (ml equ. ind } .^{-1}\right)\end{array}$ & $\begin{array}{c}\text { PGP } \\
\left(1 \text { equ. } \mathrm{m}^{-2}\right)\end{array}$ & $\begin{array}{l}\text { Density } \\
\text { (ind. } \mathrm{m}^{-2} \text { ) }\end{array}$ & $\begin{array}{l}\text { Gut pigment } \\
\text { (ml equ. ind }{ }^{-1} \text { ) }\end{array}$ & $\begin{array}{c}\text { PGP } \\
\left(1 \text { equ. } \mathrm{m}^{-2}\right)\end{array}$ \\
\hline Small copepods & 37400 & 0.08 & 3.00 & 30800 & 0.37 & 0.40 \\
\hline Oncaea spp. & 12900 & 0.07 & 0.90 & 11200 & 0.05 & 0.56 \\
\hline Copepod nauplii & 6760 & $(0.04)^{\mathrm{a}}$ & 0.27 & 9520 & 0.04 & 0.38 \\
\hline Calanus pacificus, $\mathrm{F}$ & 1160 & 2.38 & 2.76 & 2780 & 7.68 & 21.35 \\
\hline Clausocalanus spp., F & 12600 & 0.05 & 0.63 & 6700 & 0.32 & 2.14 \\
\hline Eucalanus californica, $\mathrm{F}$ & 400 & 19.00 & 7.60 & 730 & 27.20 & 19.86 \\
\hline Pleuromamma borealis, $\mathrm{F}$ & 0 & - & - & 1280 & 4.00 & 5.12 \\
\hline Pleuromamma gracilis, $\mathrm{C} 5 / \mathrm{F}$ & 0 & - & - & 87 & 9.76 & 0.85 \\
\hline Rhincalanus nasutus, $\mathrm{F}$ & 500 & 7.52 & 3.76 & 600 & 6.21 & 3.73 \\
\hline Large calanoids $>1.5 \mathrm{~mm}$ & 2610 & 1.47 & 3.84 & 3790 & 2.19 & 8.30 \\
\hline Euphausiids, calyp & 2680 & 0.20 & 0.54 & 1300 & 0.13 & 0.17 \\
\hline$<10 \mathrm{~mm}$ & 865 & 1.40 & 1.21 & 1600 & 1.94 & 3.10 \\
\hline $10-20 \mathrm{~mm}$ & 5 & $(25.00)^{a}$ & 0.12 & 66 & 25.00 & 1.65 \\
\hline Ostracods $<1.5 \mathrm{~mm}$ & 810 & 0.88 & 0.71 & 490 & 0.67 & 0.33 \\
\hline$>1.5 \mathrm{~mm}$ & 10 & $(5.46)^{a}$ & 0.05 & 29 & 5.46 & 0.16 \\
\hline Pteropods & 260 & 16.30 & 4.24 & 260 & 66.10 & 17.19 \\
\hline Thaliaceans $<2 \mathrm{~mm}$ & 9710 & 2.22 & 21.56 & 13800 & 0.17 & 2.35 \\
\hline $2-5 \mathrm{~mm}$ & 640 & 10.50 & 6.72 & 1830 & 10.89 & 19.93 \\
\hline$>5 \mathrm{~mm}$ & 610 & 24.60 & 15.01 & 1040 & 18.54 & 19.28 \\
\hline Appendicularians $<0.5 \mathrm{~mm}$ & 12800 & 0.58 & 7.42 & 15300 & 0.82 & 12.55 \\
\hline $0.5-2 \mathrm{~mm}$ & 10700 & 2.23 & 23.86 & 6730 & 6.58 & 44.28 \\
\hline Community gut pigment & & & 104.2 & & & 183.6 \\
\hline
\end{tabular}

concentration up to about $0.8 \mathrm{mg} \mathrm{chl} \mathrm{a} \mathrm{m}^{-3}$ and leveled off at an average of $7 \mathrm{ng}$ pigment ind. ${ }^{-1}$ at higher concentrations (May 1986). Other zooplankton taxa varied substantially in their gut pigment response to watercolumn chlorophyll. Among the migrators, for example, cruise-averaged values of gut fluorescence for Metridia lucens varied within a factor of 2 , but showed little indication of a food concentration effect over a factor of 20 range in chlorophyll concentration (Fig. 7). The larger migrating copepods, adult females of Eucalanus californicus, Pleuromamma borealis and $P$. abdominalis, on the other hand, did not display a strong difference in gut fluorescence with chlorophyll concentration among winter and spring cruises, although median values for these cruises generally exceeded those for the autumn cruises by a factor of 2 or 3 (Fig. 7).

Among nonmigrating taxa, Oncaea spp. showed a strong increase $\left(\mathrm{r}^{2}=0.77\right.$, linear regression) in gut fluorescence with chlorophyll concentration, and the gut pigment contents of Oikopleura spp. were highest when chlorophyll was most abundant in May 1986 (Fig. 8). During the same cruise, nocturnal gut fluorescence for combined Clausocalanus spp. females was $0.82 \mathrm{ng}$ pigment ind ${ }^{-1}(\mathrm{SD}=0.34, \mathrm{n}=4$ ), substantially higher than the mean level for either species when chlorophyll was below $1 \mathrm{mg} \mathrm{m}^{-3}$.

\section{Community gut pigment}

Daytime and nightime estimates of gut pigment content for the zooplankton community are given in Tables 1 to 6 . Gaps in the data were filled from estimates made on other cruises or times of day as noted in the table legends. Except for the first cruise (October 1985) which had many of these entries, the data collected during a given cruise generally accounted for

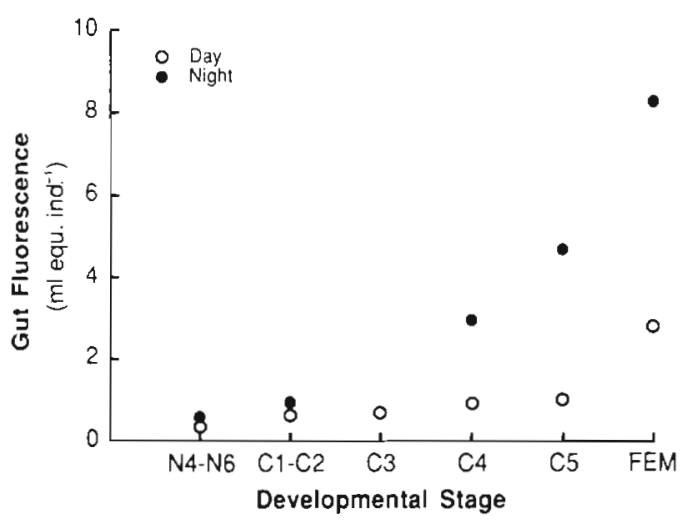

Fig. 5. Calanus pacificus. Comparison of gut pigment contents among individual developmental stages (nauplii, copepods, adult females) sampled during the day (o) and at night $(\bullet$ in April 1987 

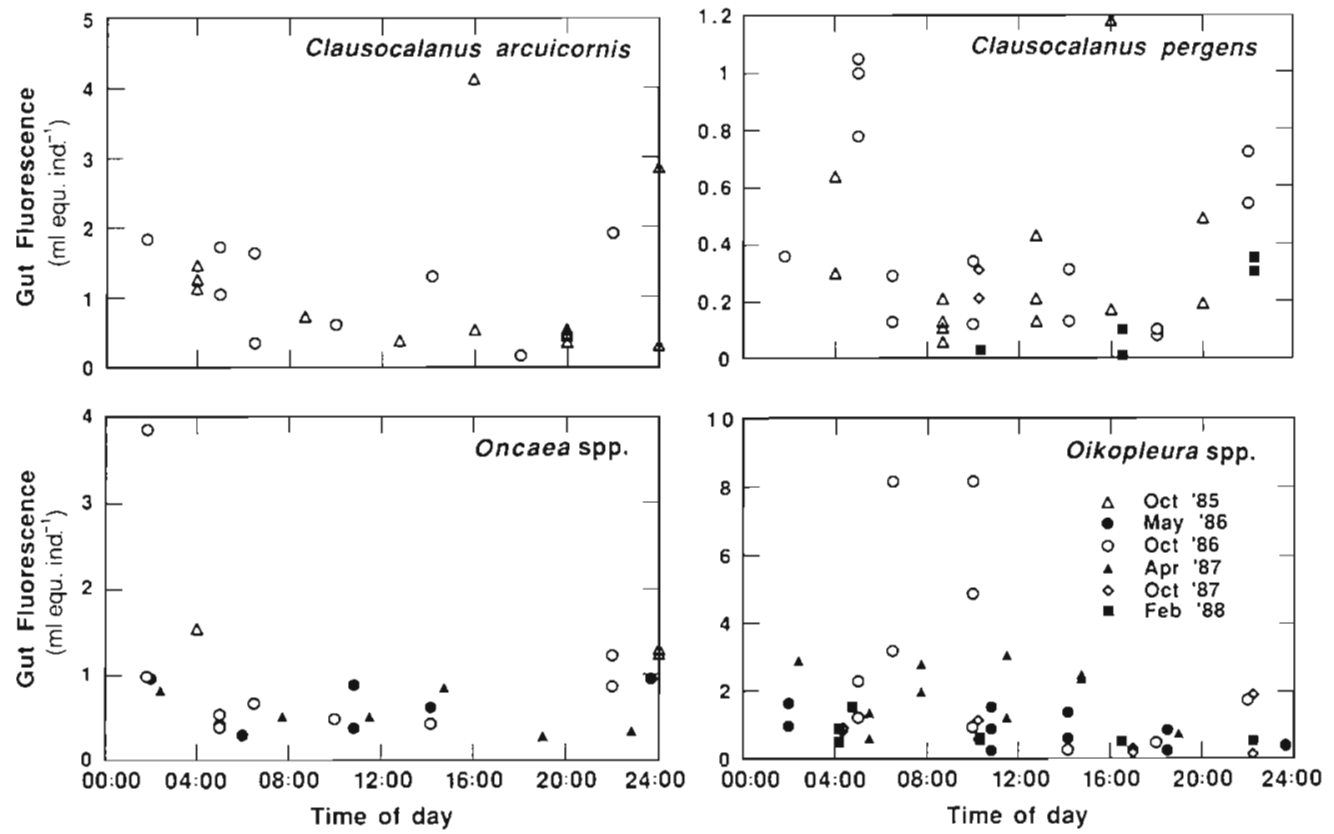

Fig. 6. Clausocalanus, Oncaea and Oikopleura spp. Diel patterns in gut pigment contents of small mesozooplankton species in the Santa Monica Basin

$90 \%$ or more of the community estimate of gut pigment content for that cruise.

Community gut pigment (CGP) varied by about an order of magnitude over all cruises. The lowest cruiseaveraged value was equivalent to 45.7 l of watercolumn chlorophyll cleared $\mathrm{m}^{-2}$ (October 1987, day) the highest was 321 l equ. $\mathrm{m}^{-2}$ (April 1987, night). These instantaneous 'standing stocks' of gut pigment correspond, respectively, to 0.065 and $0.46 \%$ of depthintegrated chlorophyll in the upper $70 \mathrm{~m}$. Highest daytime values of CGP (as equ. volume cleared) occurred in late winter and early spring (February and
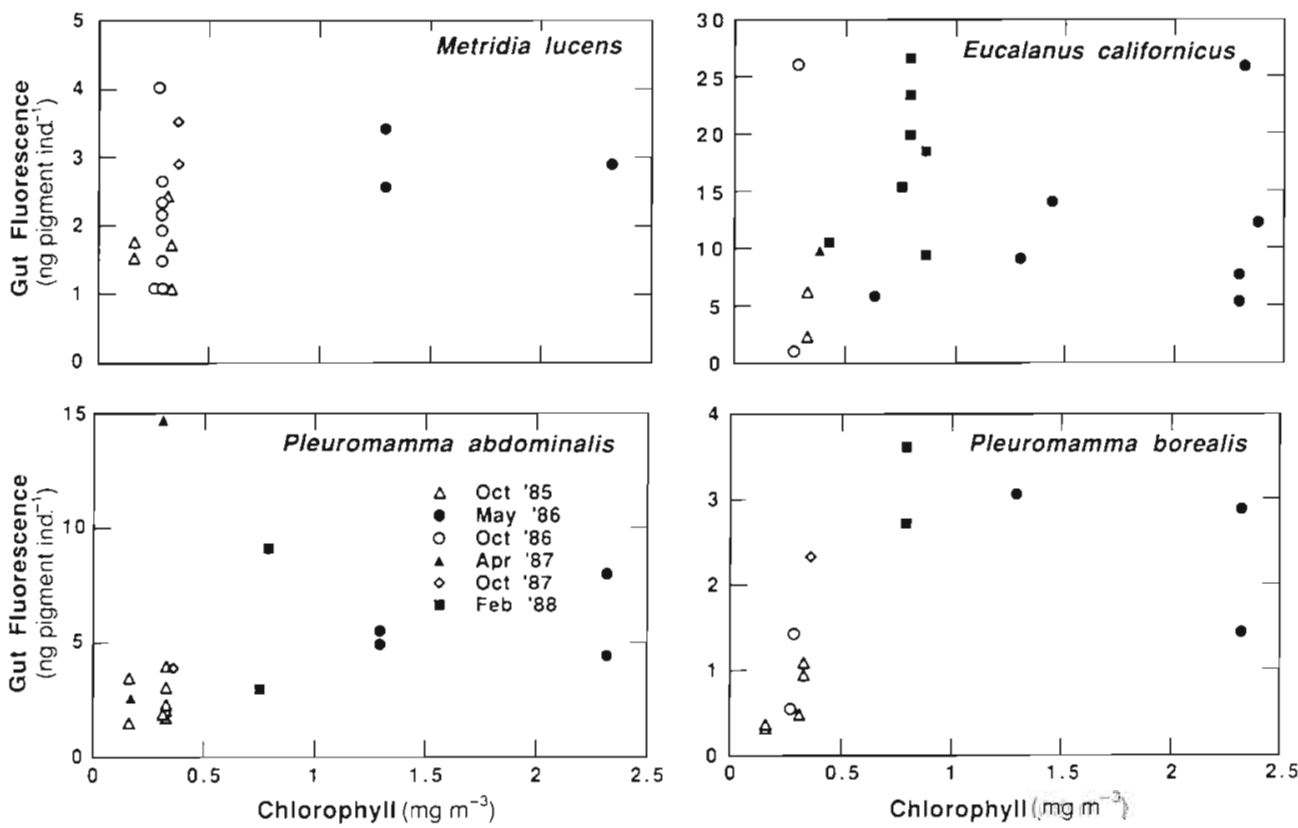

Fig. 7. Metridia lucens, Eucalanus californicus and Pleuromamma spp. Relationships between nighttime gut pigment contents and mean euphotic zone chlorophyll concentration for large migrating copepod species in the Santa Monica Basin. All specimens were adult females 

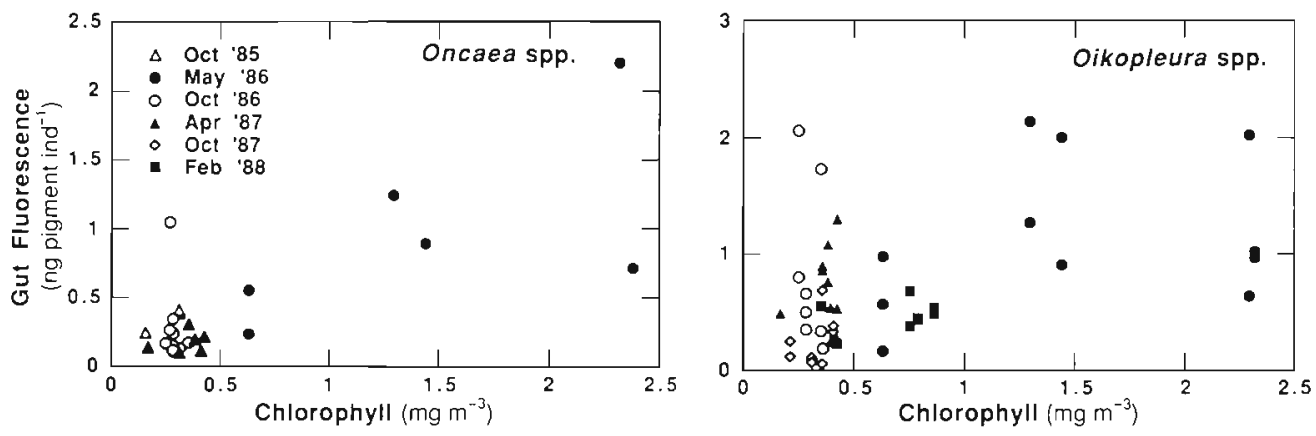

Fig. 8. Oncaea and Oikopleura spp. Relationships between gut pigment contents and mean euphotic zone chlorophyll concentration for small mesozooplankton in the Santa Monica Basin

April) and were up to 2 to 3 times higher than the values for other months. High nighttime pigment contents also occurred in April 1987 (321 l equ. $\mathrm{m}^{-2}$ ). Even though May 1986 appears below average among the cruises in terms of volume-equivalent pigment content for the mesozooplankton community, it ranks first as

Table 7. Night:day ratio of community gut pigment (CGP) and contribution of migrating taxa to nighttime CGP for mesozooplankton of Santa Monica Basin

\begin{tabular}{|lcc|}
\hline Cruise & $\begin{array}{c}\text { CGP ratio } \\
\text { (night:day) }\end{array}$ & $\begin{array}{c}\text { Migrators } \\
\text { (\% CGP) }\end{array}$ \\
\hline Winter-spring & & \\
Feb 1988 & 1.76 & 18.8 \\
Apr 1987 & 1.95 & 47.2 \\
May 1986 & 1.02 & 10.3 \\
Autumn & & \\
Oct 1985 & 1.77 & 12.1 \\
Oct 1986 & 2.70 & 9.6 \\
Oct 1987 & 3.39 & 22.8 \\
\hline
\end{tabular}

CGP (ng pigment $\mathrm{m}^{-2}$ ) due to the high water-column chlorophyll during the cruise.

CGP showed the highest diel difference in the autumn, the ratio of night: day CGP varying from 1.8 to 3.4 for the 3 October cruises (Table 7). Night CGP only marginally exceeded day values for the May cruise. The contribution of migrating taxa to nighttime CGP varied from 10 to $47 \%$ without a seasonal trend (Table 7). Generally, migrators contributed less than $20 \%$ of nighttime CGP. However, migrators could account for the diel difference in CGP for April 1987 when the night:day ratio was about 2 , and half of the night CGP was due to migrators. From similar comparisons, migrators explained one-third or less of the diel difference during other times of the year when daynight differences appeared to exist. In October 1986 as an extreme, only about $5 \%$ of the diel difference could be attributed to migrators.

The distibutions of CGP among major taxonomic groups and between smaller and larger individuals are presented in Tables $8 \& 9$. Copepods accounted for the majority of CGP in winter-spring, with the exception of

Table 8. Seasonal comparison of community gut pigment (CGP) among different taxa of mesozooplankton in the Santa Monica Basin. D: day; N: night

\begin{tabular}{|c|c|c|c|c|c|c|c|c|}
\hline \multirow{2}{*}{\multicolumn{2}{|c|}{ Cruise }} & \multicolumn{7}{|c|}{ Taxa (\% CGP) } \\
\hline & & Copepod & Euphausiid & Ostracod & Cladocera & Pteropod & Salp & Appendicularia \\
\hline \multicolumn{9}{|c|}{ Winter-spring } \\
\hline \multirow{2}{*}{ Feb $1988^{\circ}$} & $D$ & 21.9 & 1.8 & 0.7 & 0.0 & 2.9 & 41.5 & 30.1 \\
\hline & $\mathrm{N}$ & 34.2 & 2.6 & 0.2 & 0.0 & 9.4 & 22.6 & 30.9 \\
\hline \multirow[t]{2}{*}{ Apr 1987} & $\mathrm{D}$ & 56.9 & 0.8 & 0.4 & 1.2 & 0.0 & 6.4 & 34.2 \\
\hline & $\mathrm{N}$ & 79.1 & 2.4 & 0.4 & 0.7 & 0.0 & 2.1 & 14.6 \\
\hline \multirow[t]{2}{*}{ May 1986} & $\mathrm{D}$ & 48.6 & 7.2 & 0.1 & 0.0 & 0.0 & 8.1 & 36.2 \\
\hline & $\mathrm{N}$ & 54.6 & 11.2 & 2.0 & 0.0 & 0.0 & 6.5 & 25.7 \\
\hline \multicolumn{9}{|l|}{ Autumn } \\
\hline \multirow[t]{2}{*}{ Oct 1985} & $\mathrm{D}$ & 35.7 & 0.7 & 0.0 & 0.0 & 27.7 & 1.1 & 34.8 \\
\hline & $\mathrm{N}$ & 40.2 & 1.9 & 4.4 & 0.0 & 8.6 & 0.6 & 44.4 \\
\hline \multirow[t]{2}{*}{ Oct 1986} & $\mathrm{D}$ & 51.2 & 0.1 & 0.0 & 0.0 & 0.1 & 0.4 & 48.1 \\
\hline & $\mathrm{N}$ & 57.3 & 0.4 & 2.8 & 0.0 & 0.1 & 1.8 & 37.5 \\
\hline \multirow[t]{2}{*}{ Oct 1987} & $\mathrm{D}$ & 26.7 & 1.7 & 0.2 & 0.0 & 1.1 & 2.6 & 67.7 \\
\hline & $\mathrm{N}$ & 443 & 4.0 & 6.8 & 0.0 & 1.0 & 1.1 & 44.6 \\
\hline
\end{tabular}


Table 9. Contribution of small taxa ( $<1.5 \mathrm{~mm}$ body length) to community gut pigment (CGP) of mesozooplankton in Santa Monica Basin. Analyses are from vertical plankton tows $(70 \mathrm{~m}$ to surface) during day and night

\begin{tabular}{|lcc|}
\hline Cruise & Day $(\%$ CGP $)$ & Night $(\%$ CGP) \\
\hline Winter-spring & & \\
Feb 1988 & 56.5 & 33.2 \\
Apr 1987 & 76.9 & 41.6 \\
May 1986 & 61.5 & 53.7 \\
Autumn & & \\
Oct 1985 & 99.8 & 87.1 \\
Oct 1986 & 98.7 & 96.2 \\
Oct 1987 & 95.1 & 63.6 \\
\hline
\end{tabular}

February 1988, during which copepod gut pigment was about comparable to that of both thaliaceans and appendicularians (Table 8 ). Copepods generally represented less than $50 \%$ of CGP in the autumn (October) period, when appendicularians were more important. Since copepods were better represented among the migrating taxa, their contribution to CGP usually increased at night. Except for the February cruise when salps predominated and October 1985 when pteropods were important, the combined gut pigment contents of copepods and appendicularians accounted for 80 to $90 \%$ of CGP. Among the other taxa, euphausiids tended to be seasonally more important in the spring, ostracods during the autumn at night, and cladocerans and salps in the winter and spring. Regardless of seasonal differences in breakdown of CGP among grazing taxa, small forms (nominally animals $<1.5 \mathrm{~mm}$ length, but thaliaceans up to $2 \mathrm{~mm}$ were included in this category when not resolved to smaller size) were generally most important (Table 9). Only for nighttime samples on the February and April cruises, when migrators were particularly abundant, did larger taxa account for substantially more than half of CGP. Small grazers were particularly important in the autumn, exceeding $95 \%$ of daytime CGP on each of 3 October cruises and ranging from 64 to $96 \%$ of CGP at night.

\section{DISCUSSION}

\section{Gut pigment estimates}

The gut fluorescence method has become a popular approach for studying zooplankton grazing on phytoplankton under field conditions, and the present study extends the numbers of species and groups (e.g. pteropods and appendicularians) to which this approach has been applied. Where comparisons can be made, our estimates are about in the middle of those from previ- ous studies. For instance, our values of 6 to $10 \mathrm{ng}$ pigment ind.$^{-1}$ for female Calanus pacificus under high chlorophyll conditions in May 1986 compare favorably with estimates of 8 to $14 \mathrm{ng}$ ind $^{-1}$ (May 1986) and 4 to $8 \mathrm{ng}$ ind. $^{-1}$ (August 1986) for the same species in Dabob Bay, Washington, USA (Dagg et al. 1989). However, they are lower than other estimates off California which range as high as 50 to $80 \mathrm{ng}$ pigment ind. $^{-1}$ (Kleppel \& Pieper 1984, Ohman 1988). Gut pigments of 30 to $50 \mathrm{ng}$ ind. ${ }^{-1}$ have been observed for C. pacificus females when the copepods are actively feeding on dense concentrations of cultured algae in the laboratory, but even then median or steady-state values generally lie at or below $10 \mathrm{ng}$ ind ${ }^{-1}$ (Mackas \& Burns 1986, Mobley 1987, Lopez et al. 1988, see also Ellis \& Small 1989 for C. marshallae). Estimates of 1 to $8 \mathrm{ng}$ ind. ${ }^{-1}$ are typical of values reported for the nighttime feeding of comparably sized congeners, $C$. chilensis, C. finmarchicus and C. helgolandicus under field conditions (Boyd et al. 1980, Baars \& Oosterhuis 1984, Simard et al. 1985, Harris \& Malej 1986, Morales et al. 1993). Higher values have been reported, however, for female $C$. finmarchicus during spring bloom conditions (2 to $24 \mathrm{ng}$ ind. ${ }^{-1}$ for the Barents Sea, Tande \& Båmstedt $1985 ; 13$ to $35 \mathrm{ng}$ ind.$^{-1}$ for the New York Bight, Smith \& Lane 1988).

Similar comparisons can be made between the present estimates of gut pigments for female Metridia lucens ( $=M$. pacifica, Dagg et al. 1989) and those of previous studies. Our high values of 3 to $4 \mathrm{ng}_{\text {ind. }}{ }^{-1}$ are about half of the steady state pigment contents observed under high-food laboratory conditions at $12^{\circ} \mathrm{C}$ (Mackas \& Burns 1986) and in surface waters of Dabob Bay (Dagg et al. 1989). Batchelder's (1986) mean nighttime estimates of about $6 \mathrm{ng}$ ind.$^{-1}$ are in the range that we observed after correction for the factor of 1.5 applied to his pigment data (e.g. Conover et al. 1986). Smith \& Lane's (1988) estimates of 11 to $46 \mathrm{ng}$ ind.$^{-1}$ are still well beyond our values, however, and surprisingly exceed, by a factor of 2 or 3 in some cases, the gut pigments for the much larger Calanus finmarchicus collected at the same time.

Some of the disparity in various estimates of gut pigment may be due to methodological differences, in particular the handling of animals in the critical first few minutes after capture. We did not narcotize our collections (Kleppel \& Pieper 1984) or freeze them in liquid nitrogen (Ohman 1988). However, we did routinely get the animals from the net codends onto Nitex screening and into the freezer within 5 min of the start of the net tow, so it is not likely that handling or sorting delays caused our estimates to be low by very much. Our lower mean values of gut pigments could be explained in part by having sampled over a broad depth range including large variations in chlorophyll 
concentration rather than a zone of concentrated chlorophyll. The vertical distribution of zooplankton biomass covaries with chlorophyll concentration in the southern California Bight, but the relationship is weak and variable among species and developmental stages (Mullin \& Brooks 1972, Napp et al. 1988). Napp et al. (1988), for instance, showed no significant relationship between nighttime distributions of large migratory copepods (including Calanus spp. and Metridia lucens females) and chlorophyll. Another factor is the timing of our collections, which generally missed the period immediately after sunset when the gut contents of migratory animals are typically highest (e.g. Ohman 1988). In this regard, since Calanus spp. females were not obviously migrating during the May 1986 period of high chlorophyll, it is not suprising that their gut pigments were more typical of the mean values for copepods maintained in the laboratory at constant high food rather than the exaggerated levels of animals given food after a period of starvation. In addition to the above-mentioned factors, differences in the gut pigment contents of zooplankton from different studies or circumstances must be interpreted in the context of potential losses of chl a and its by-products during digestion and the rate of gastric evacuation. When these factors are considered (Landry et al. 1994), the clearance rates calculated from our estimates of gut pigment contents are quite reasonable relative to the known capabilities of well-studied taxa like Calanus.

\section{Relationships to food concentration}

The dangers in overinterpreting gut pigment data can be illustrated by considering the patterns in variability with chlorophyll concentration for the combined 6 cruises. The data for Calanus pacificus females (Fig. 4b), for instance, look remarkably like a textbook functional response; hence it is tempting to speculate that $C$. pacificus is food limited when mean chlorophyll concentration in the upper $70 \mathrm{~m}$ falls below $0.7 \mathrm{mg} \mathrm{m}^{-3}$. Egg production, a more appropriate index of food limitation, for C. pacificus females also has shown a marked hyperbolic dependency on chlorophyll concentration (Runge 1985). Nonetheless, the relationship between grazing rate and food concentration is confounded by uncertainties in the distributions of grazers and concentrations of food and possible seasonal differences in pigment destruction and gut evacuation rates. In addition, since virtually all of the organisms that we investigated are either omnivorous (e.g. see Kleppel et al. 1988 for evidence of ingested animal carotenoid for Calanus spp., Clausocalanus spp., and Evadne spp. in the Southern California Bight) or can exploit food sources (bacteria, protozoans, detritus) which lack chlorophyll, lower levels of gut pigments do not necessarily mean lower amounts of total food available or ingested.

Seasonal variations in organism size must also be considered in interpreting gut pigment patterns. Assuming that copepod size followed the usual inverse relationship with environmental temperature in our study, Fig. 4 implicitly compares larger animals, with larger gut capacity and feeding potential, in winter and spring cruises with smaller animals in autumn. In principle, differences in the size distributions of appendicularians (Fig. 3) could explain higher gut pigment content in spring cruises as compared to autumn (King et al. 1980, Alldredge 1981). However, size does not account for the relationship between gut pigment and chlorophyll when only spring and winter cruises are considered (Fig. 8).

\section{Diel patterns}

Since the original description of the method (Mackas \& Bohrer 1976), gut fluorescence from chlorophyll and its by-products has been used extensively to document the diel feeding activities of planktonic herbivores (e.g. Boyd et al. 1980, Nicolajsen et al. 1983, Baars \& Oosterhuis 1984, Dagg 1985, Head et al. 1985, Simard et al. 1985, Head \& Harris 1987 , Peterson et al. 1990, Stuart \& Pillar 1990, Runge \& Ingram 1991, Morales et al. 1993). Bimodal patterns in gut fluorescence, implying highest nocturnal feeding activity after sunset and before sunrise with a midnight period of reduced feeding, have been demonstrated for various species of Calanus, Acartia, Pseudocalanus, and Neocalanus (Simard et al. 1985, Head \& Harris 1987, Ishii 1990). Ohman (1988) has provided evidence of bimodal feeding for adult and C5s of Calanus pacificus and Rhincalanus nasutus off Central California, noting that the diel pattern in feeding activity explained more of the variability in copepod gut fluorescence in this area than horizontal variations in food supply. The present study focuses on broad community-level patterns rather than the details of individual species; hence, we did not attempt to sample at temporal and spatial scales sufficient to resolve the intricacies of diel behaviors, which are clearly more complicated than simple day-night differences.

Relatively little of the day-night differences in CGP in the present study can be linked to the migratory behaviors of organisms (Metridia lucens, Pleuromamma spp., adult euphausiids and large ostracods) that unambiguously leave the top $70 \mathrm{~m}$ of the water column during the day and return at night. Some of the larger 
copepods, e.g. Eucalanus californicus and Rhincalanus nasutus, showed little evidence of pronounced migration but substantial day-night difference in feeding activity (e.g. $R$. nasutus in April 1987). Among the more abundant large species, migratory behavior of Calanus pacificus varied seasonally. C. pacificus females and C5s did not leave the euphotic zone during the day or exhibit enhanced nocturnal feeding in May 1986. However, diel differences in gut pigment content were abserved earlier and later in the year (except for October 1987) when the majority of the later developmental stages appeared to reside below $70 \mathrm{~m}$ during the day. Indeed, the combined effect of higher nocturnal abundances and individual gut pigments of $C$. pacificus in April 1987 accounted for most of the large day-night difference in CGP during this cruise.

Our estimates of gut pigments for the mesozooplankton community overlying Santa Monica Basin suggest that day-night differences in grazing intensity were greatest when chlorophyll concentration in the water column was low (April 1987 and October 1985, 1986, and 1987; Table 7). This result is consistent with the observations of Boyd et al. (1980) and Nicolajsen et al. (1983) for different zooplankton communities sampled spatially and seasonally, and it may represent a general pattern. Intuitively, one would expect zooplankton to feed at a more constant rate throughout day and night when food is limiting and to exhibit diel behavior when food is above the normal saturation point. In the latter case, the starvation response (Runge 1980) would partially compensate for reduced feeding when the animals are out of the euphotic zone. Dagg (1985), for example, found that the copepod Neocalanus plumchrus migrated out of the euphotic zone on a diel cycle when food was abundant, but did not migrate when food was scarce. Boyd et al. (1980) ascribed the nonintuitive inverse trend to possible temporal partitioning of food resource among competing zooplankton under conditions of low food abundance. Nicolajsen et al. (1983) argued that this explanation did not apply to the circumstances of their study, and we likewise find unsatisfying the implication that mesozooplankton exert sufficient grazing pressure on phytoplankton to make partitioning of the resource an optimal energetic strategy. While nocturnal grazing by the larger migratory taxa explained most of the diel signal in April 1987, this was not the case for the autumn cruises. Particularly in October 1986, for which the data are most complete, diel differences in gut pigments of smaller, nonmigratory animals (Clausocalanus spp., Oncaea spp., and small appendicularians) accounted for most $(78 \%)$ of the observed difference in day and night CGP. These results imply that there is a behavioral component to the diel feeding pattern which is not linked to vertical migration. Such behaviors are well documented experimentally in the laboratory and the field, even under food-limiting conditions (Dam 1986, Head 1986, Stearns 1986, Durbin et al. 1990). The present study cannot distinguish between behavioral explanations of feeding periodicities and the possibility that subtle shifts in distributions within the upper $70 \mathrm{~m}$ of the water column cause smaller taxa to experience significant variations in the relative abundance of food on a diel cycle (e.g. Mullin \& Brooks 1976).

\section{Community trends}

The present study yields 3 general conclusions about the partitioning of mesozooplankton grazing on phytoplankton in the water column overlying Santa Monica Basin. First, small, nonmigratory zooplankton are generally more important grazers than larger, migratory forms. Second, gelatinous taxa, specifically small pelagic tunicates, contribute significantly to grazing and may frequently be more important than crustaceans. Third, the size structure of the community varies seasonally such that larger taxa are relatively more important in the late winter and spring while smaller forms are particularly dominant in autumn.

Our results for the size structure of the grazing community are in good qualitative agreement with recent studies based on gut pigment analyses of mechanically size-fractioned zooplankton communities. Tsuda et al. (1989) found that microzooplankton dominated grazing in the subtropical North Pacific; though less important overall, smaller net-collect mesozooplankton ( 95 to $350 \mu \mathrm{m}$ ) accounted for about double the grazing rates of larger forms. Similarly, Morales et al. (1991, 1993) found that the smallest (200 to $500 \mu \mathrm{m})$ of 3 size fractions was generally the most important, accounting for at least $35 \%$ and generally dominating copepod grazing at stations in the Northeast Atlantic. Methodological differences preclude a strict quantitative comparison among these studies and our own. Nonetheless, that similar conclusions can be reached from studies in very different ocean environments suggests that small species and developmental stages of planktonic animals are generally the most important primary consumers within the mesozooplankton. This assumes of course, that net avoidance by larger and faster swimming zooplankton (e.g euphausiids) does not seriously bias the assessment of relative grazer biomass and gut pigment content. In the present study, this potential bias is at least partially offset by the fact that we did not acount for the grazing of nauplii and other abundant and extremely small animals (e.g. 
Microsetella spp.), most of which passed through our relatively coarse mesh net.

Most field studies of zooplankton feeding deal with specific developmental stages, a given species or, at best, a taxonomically defined subset of the grazer assemblage. Thus, the relative contributions of species and groups to community grazing is generally left vague. Alldredge (1981), for instance, studied the feeding rates of appendicularians in the Gulf of California and concluded that they contribute significantly to grazing pressure on phytoplankton, with individual species clearing up to $38 \%$ of near surface water per day. The present study indicates that appendicularians are indeed an important component of the grazing community off Southern California, and may in fact be more important than copepods for at least part of the year. In contrast to the relatively constant high abundance and activity of appendicularians, salps and pteropods are more episodic in their appearance and importance as grazers (e.g. Bathmann 1988, Tsuda \& Nemoto 1992). During such 'blooms' (e.g. February 1988), the combined gut pigment content of the gelatinous zooplankton can swamp that of planktonic crustaceans (Table 8).

The shift in the size structure of the mesozooplankton community from larger animals in winter and spring to smaller forms in late summer and autumn is typical of many temperate systems and reflects the seasonal change from dominance of abundant large diatoms early in the year to small flagellates and picoplankton during summer stratification (e.g. Malone 1971, Landry 1977, Steele \& Frost 1977). Smaller mesozooplankton are likely to be more similar functionally to microzooplankton than to larger mesozooplankton with regard to the remineralization of their fecal debris within the euphotic zone (e.g. Paffenhöfer \& Knowles 1979). Therefore, a seasonal change in the ratio of fecal pellet flux to mesozooplankton grazing would be expected to follow the shift in size structure of the community. The relationships among mesozooplankton size structure, grazing pressure, and particle flux are examined in detail in a companion paper (Landry et al. 1994).

Acknowledgements. The authors gratefully acknowledge the exceptional efforts and positive attitudes of the captain, crew, and marine techs of the RV 'New Horizon', D. Ripley who provided technical assistance on one of the cruises, and J. Fujimoto who prepared the figures and text. This study was supported by Grants DE-F605-85 ER60335 and -88ER60628 from the U.S. Department of Energy and prepared for publication under National Science Foundation Grant OCE9218152. Contribution No. 3638 from the School of Ocean and Earth Science and Technology, University of Hawaii at Manoa, Honolulu, HI 96822, USA

\section{LITERATURE CITED}

Alldredge, A. L. (1981). The impact of appendicularian grazing on natural food concentrations in situ. Limnol. Oceanogr. 26: 247-257

Azam, F., Fenchel, T., Field, J. G., Gray, J. S., Meyer-Reil, L. A., Thingstad, F. (1983). The ecological role of water-column microbes in the sea. Mar. Ecol. Prog. Ser. 10:257-263

Baars, M. A., Oosterhuis, S. S. (1984). Diurnal feeding rhythms in North Sea copepods measured by gut fluorescence digestive enzyme activity and grazing on labelled food. Neth. J. Sea Res. 18: 97-119

Batchelder, H. P. (1986). Phytoplankton balance in the oceanic subarctic Pacific: grazing impact of Metridia pacifica. Mar. Ecol. Prog. Ser. 34: 213-225

Bathmann, U. V. (1988). Mass occurrence of Salpa fusiformis in the spring of 1984 off Ireland: implications for sedimentation processes. Mar. Biol. 97: 127-135

Bedo, A. W., Acuna, J. L., Robins, D., Harris, R. P. (1993). Grazing in the micron and the sub-micon particle size range: the case of Oikopleura dioica (Appendicularia). Bull. mar. Sci. 53: 2-14

Boyd, C. M., Smith, S. L., Cowles, T. J. (1980). Grazing patterns of copepods in the upwelling system off Peru. Limnol. Oceanogr. 25: 583-596

Capriulo, G. M., Carpenter, E. J. (1980). Grazing by 35 to $202 \mu \mathrm{m}$ microzooplankton in Long Island Sound. Mar. Biol. 56: 319-326

Conover, R. J., Durvasula, R., Roy, S., Wang, R. (1986). Probable loss of chlorophyll-derived pigments during passage through the gut of zooplankton, and some of the consequences. Limnol. Oceanogr. 31: 878-887

Dagg, M. J (1985). The effects of food limitation on diel migratory behavior in marine zooplankton. Arch. Hydrobiol. 21: 247-255

Dagg, M. J., Frost, B. W., Walser, W. E. Jr (1989). Copepod diel migration, feeding, and the vertical flux of phaeopigments. Limnol. Oceanogr. 34: 1062-1071

Dam, H. G. (1986). Short-term feeding of Temora longicornis Müller in the laboratory and the field. J. exp. mar. Biol. Ecol. 99: 149-161

Durbin, A. G., Durbin, E. G., Wlodarczyk, E. (1990). Diel feeding behavior in the marine copepod Acartia tonsa in relation to food availability. Mar. Ecol. Prog. Ser. 68: $23-45$

Ellis, S. G., Snall, L. F. (1989). Comparison of gut-evacuation rates of feeding and non-feeding Calanus marshallae. Mar. Biol. 103: 175-181

Fasham, M. J. E. (1985). Flow analysis of materials in the marine euphotic zone. Can. Bull. Fish. Aquat. Sci. 213: $139-162$

Frost, B. W. (1984). Utilization of phytoplankton production in the surface layer. In: Global ocean flux study: proceedings of a workshop, September 10-14, 1984. National Academy Press, Washington, DC, p. 125-134

Hansen, B., Tande, K. S., Berggreen, U. C. (1990). On the trophic fate of Phaeocystis pouchetii (Harlot). III. Functional responses in grazing demonstrated on juvenile stages of Calanus finmarchus (Copepoda) fed diatoms and Phaeocystis. J. Plankton Res. 12: 1173-1187

Harbison, G. R., Gilmer, R. W. (1976). The feeding rates of the pelagic tunicate, Pegea confoederata, and two other salps. Limnol. Oceanogr. 21:517-528

Harris, R. P. Malej, A. (1986). Diel patterns of ammonium excretion and grazing rhythms in Calanus helgolandicus in surface stratified waters. Mar. Ecol. Prog. Ser. 31: $75-85$ 
Head, E. J. H. (1986). Estimation of Arctic copepod grazing rates in vivo and comparison with in vitro methods. Mar. Biol. 92: 371-379

Head, E. J. H., Harris, L. R. (1987). Copepod feeding patterns before and during a spring bloom in Bedford Basin, Nova Scotia. Mar. Ecol. Prog. Ser. 40: 221-230

Head, E. J. H., Harris, L. R., Abou Debs, C. (1985). Effect of daylength and food concentration on in situ diurnal feeding rhythms in Arctic copepods. Mar. Ecol. Prog. Ser. 24: $281-288$

Hickey, B. (1992). Circulation over the Santa Monica-San Pedro Basin and shelf. Prog. Oceanogr. 30: 37-115

Holm-Hansen, O., Lorenzen, C. J., Holmes, R. W., Strickland, J. D. (1965). Fluorometric determination of chlorophyll. J. Cons. int. Explor. Mer 30: 3-15

Ishii, H. (1990). In situ feeding rhythms of herbivorous copepods, and the effect of starvation. Mar. Biol. 105: 91-98

King, K. R, Hollibaugh, J. T., Azam, F. (1980). Predator-prey interactions between larvacean Oikopleura dioica and bacterioplankton in enclosed water columns. Mar. Biol. 56: $49-57$

Kleppel, G. S., Frazel, D., Pieper, R. E., Holliday, D. V. (1988). Natural diets of zooplankton off southern California. Mar. Ecol. Prog. Ser. 49: 231-241

Kleppel, G. S., Pieper, R. E. (1984). Phytoplankton pigments in the gut contents of planktonic copepods from coastal waters off southern California. Mar. Biol. 78: 193-198

Landry, M. R. (1977). A review of important concepts in the trophic organization of pelagic ecosystems. Helgoländer wiss. Meeresunters. 30: 8-17

Landry, M. R., Hassett, R. P. (1982). Estimating the grazing impact of marine micro-zooplankton. Mar. Biol. 67: 283-288

Landry, M. R., Lorenzen, C. J., Peterson, W. K. (1994). Mesozooplankton grazing in the Southern California Bight. I1. Grazing impact and particulate flux. Mar. Ecol. Prog. Ser. 115: $73-85$

Lopez, M. D. G., Huntley, M. E., Sykes, P. F. (1988). Pigment destruction by Calanus pacificus: impact on the estimation of water column fluxes. J. Plankton Res. 10: 715-734

Mackas, D., Bohrer, R. (1976). Fluorescence analysis of zooplankton gut contents and an investigation of diel feeding patterns. J. exp. mar. Biol. Ecol. 25: 77-85

Mackas, D. L., Burns, K. E. (1986). Poststarvation feeding and swimming activity in Calanus pacificus and Metridia pacifica. Limnol. Oceanogr. 31: 383-392

Madin, L. P. (1974). Field observations on the feeding behavior of salps (Tunicata: Thaliacea). Mar. Biol 25: 143-147

Malone, T C. (1971). The relative importance of nanoplankton and netplankton as primary producers in the California Current system. Fish. Bull. U.S. 69: 799-820

Michaels, A. F., Silver, M. W. (1988). Primary production sinking fluxes and the microbial food web. Deep Sea Res 35: $473-490$

Mobley, C. T. (1987). Time-series ingestion rate estimates on individual Calanus pacificus Brodsky: interactions with environmental and biological factors. J. exp. mar. Biol. Ecol. 114: 199-216

Morales, C. E., Bedo, A., Harris, R. P., Tranter, P. R. G. (1991) Grazing of copepod assemblages in the north-east Atlantic: the importance of the small size fraction. J. Plankton Res. 13: 455-472

Morales, C. E., Harris, R. P., Head, R. N., Tranter, P. R. G (1993). Copepod grazing in the oceanic northeast Atlantic during a 6 week drifting station: the contribution of size classes and vertical migrants. J. Plankton Res. 15: 185-211.

Mullin, M. M. (1986). Spatial and temporal scales and patterns. In: Eppley, R. W. (ed.) Phytoplankton dynamics of the Southern California Bight. Springer-Verlag, Berlin, p. 216-273

Mullin, M. M., Brooks, E. R. (1972). The vertical distribution of juvenile Calanus (Copepoda) and phytoplankton within the upper $50 \mathrm{~m}$ of water off La Jolla, California. In: Takenouti, A. Y (ed.) Biological oceanography of the northern North Pacific Ocean. Idemitsu Shoten, Tokyo, p. 347-354

Mullin, M. M., Brooks, E. R. (1976). Some consequences of distributional heterogeneity of phytoplankton and zooplankton. Limnol. Oceanogr. 21.784-796

Napp, J. M., Brooks, E. R., Matrai, P., Mullin, M. M. (1988). Vertical distribution of marine particles and grazers. II. Relation of grazer distribution to food quality and quantity. Mar. Ecol. Prog. Ser. 50: 59-72

Nicolajsen, H., Mohlenberg, F., Kiørboe, T. (1983). Algal grazing by the planktonic copepods Centropages hamatus and

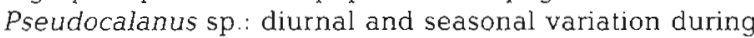
the spring phytoplankton bloom in the Oresund. Ophelia 22: $15-31$

Ohman, M. D. (1988). Sources of variability in measurements of copepod lipids and gut fluorescence in the California Current coastal zone. Mar. Ecol. Prog. Ser. 42: 143-153

Paffenhöfer, G.-A., Knowles, S. C. (1979). Ecological implications of fecal pellet size, production and consumption. J. mar. Res. 37: 35-49

Peinert, R., von Bodungen, B., Smetacek, V. S. (1989). Food web structure and loss rate. In: Berger, W. H., Smetacek, V. S., Wefer, G. (eds.) Productivity of the ocean: past and present. John Wiley and Sons, New York, p. 35-48

Peterson, W. T., Painting, S. J., Hutchings, L. (1990). Diel variations in gut pigment content, diel vertical migration and estimates of grazing impact for copepods in the southern Benguela upwelling region in October 1987 J. Plankton Res. 12: 259-281

Runge, J. A. (1980). Effects of hunger and season on the feeding behavior of Calanus pacificus. Limnol. Oceanogr. 25: $134-145$

Runge, J. A. (1985). Relationship of egg production of Calanus pacificus to seasonal changes in phytoplankton availability in Puget Sound, Washington. Limnol. Oceanogr. 30: 382-396

Runge, J. A., Ingram, R. G. (1991). Under-ice feeding and diel migration by the planktonic copepods Calanus glacialis and Pseudocalanus minutus in relation to the ice algal production cycle in southeastern Hudson Bay, Canada. Mar. Biol. 108: 217-225

Ryther, J. H. (1969). Photosynthesis and fish production in the sea. The production of organic matter and its conversion to higher forms of life vary throughout the world ocean. Science 166: 72-76

Sherr, B. F., Sherr, E. B. (1984). Role of heterotrophic protozoa in carbon and energy flow in aquatic ecosystems. In: Klugg, M. J., Reddy, C. A. (eds.) Current perspectives in microbial ecology. American Society of Microbiology, Washington, DC, p. $412-423$

Simard, Y., Lacroix, G., Legendre, L. (1985). In situ twilight grazing rhythm during diel vertical migrations of a scattering layer of Calanus finmarchicus. Limnol. Oceanogr. 30: $598-606$

Small, L. F., Landry, M. R. Eppley, R. W., Azam, F., Carlucci, A. F. (1989). Role of plankton in the carbon and nitrogen budgets of Santa Monica Basin, Caljfornia. Mar. Ecol. Prog. Ser. 56: 57-74

Smith, S. L., Lane, P. V. Z. (1988). Grazing of the spring diatom bloom in the New York Bight by the calanoid copepods Calanus finmarchicus, Metridia lucens and Centropages typicus. Cont. Shelf Res. 8: 485-509 
Stearns, D. E. (1986). Copepod grazing behavior in simulated natural light and its relation to nocturnal feeding. Mar. Ecol. Prog. Ser. 30: 65-70

Steele, J. H., Frost, B. W. (1977). The structure of plankton communities. Phil. Trans. R. Soc. Lond. 280: 485-534

Stuart, V., Pillar, S. C. (1990). Diel grazing patterns of all ontogenetic stages of Euphausia lucens and in situ predation rates on copepods in the southern Benguela upwelling region. Mar. Ecol. Prog. Ser. 64: 227-241

Tande, K. S., Båmstedt, U. (1985). Grazing rates of the copepods Calanus glacialis and $C$. finmarchicus in arctic

This article was submitted to the editor waters of the Barents Sea. Mar. Biol. 87: 251-258

Tsuda, A., Furuya, K., Nemoto, T (1989). Feeding of microand macrozooplankton at the subsurface chlorophyll maximum in the subtropical North Pacific. J. exp. mar. Biol. Ecol. 132: 41-52

Tsuda, A., Nemoto, T (1992). Distribution and growth of salps in a Kuroshio warm-core ring during summer 1987. Deep Sea Res. 39: S219-S229

Yentsch, C. S., Menzel, D. W. (1963). A method for the determination of phytoplankton chlorophyll and phaeophytin by fluorescence. Deep Sea Res. 10: 221-231

Manuscript first received: December 27, 1993

Revised version accepted: August 31, 1994 